\title{
Norois
}

Environnement, aménagement, société

$215 \mid 2010 / 2$

Impacts morphogéniques des tempêtes

\section{L'action des tempêtes sur l'érosion des dunes : les enseignements de la tempête du 10 mars 2008}

Impact of storms on dune erosion: lessons from the $10^{\text {th }}$ March 2008 storm

\section{Serge Suanez et Jean-Marie Cariolet}

\section{OpenEdition}

1 Journals

\section{Édition électronique}

URL : https://journals.openedition.org/norois/3212

DOI : 10.4000/norois.3212

ISBN : 978-2-7535-1563-5

ISSN : $1760-8546$

Éditeur

Presses universitaires de Rennes

Édition imprimée

Date de publication : 30 septembre 2010

Pagination : 77-99

ISBN : 978-2-7535-1164-4

ISSN : 0029-182X

\section{Référence électronique}

Serge Suanez et Jean-Marie Cariolet, "L'action des tempêtes sur l'érosion des dunes : les

enseignements de la tempête du 10 mars 2008 », Norois [En ligne], 215 | 2010/2, mis en ligne le 01 septembre 2012, consulté le 14 janvier 2022. URL : http://journals.openedition.org/norois/3212 ; DOI : https://doi.org/10.4000/norois.3212 


\title{
L'ACTION DES TEMPÊTES SUR L'ÉROSION DES DUNES :
}

\section{LES ENSEIGNEMENTS DE LA TEMPÊTE DU IO MARS 2008}

\author{
Serge Suanez, Jean-Marie Cariolet \\ GÉOMER - UMR 6554 CNRS LETG \\ (Université de Bretagne Occidentale), \\ Institut Universitaire Européen de la Mer, Technopôle Brest-Iroise, Place Nicolas-Copernic - 29280 PLouzAnÉ, France \\ serge.suanez@univ-brest.fr, jean-marie.cariolet@univ-brest.fr
}

\section{RÉSUMÉ}

L'analyse de l'érosion des dunes par la tempête du 10 mars 2008 a été réalisée sur deux sites du Finistère nord: la dune de la plage du Vougot (commune de Guissény) correspondant à un large massif dunaire "naturel », et la dune de la plage de Boutrouilles (commune de Kerlouan) formant un cordon dunaire plus étroit, et protégé à sa base par une structure monolithique en matériau géocomposite injectée de sédiment, appelée Stabiplage $^{\circledR}$. Sur les deux sites du Vougot et de Boutrouilles, la tempête a généré un recul maximum de la dune atteignant respectivement 6 et $3 \mathrm{~m}$. Cette érosion s'explique avant tout par la combinaison d'une forte houle et d'une marée de vive-eau (coeff. 106) qui a engendré des niveaux d'eau extrêmes à la côte dépassant de 1 à 1,80 m suivant les secteurs, l'altitude du pied de dune. Les mesures de profils de plage/dune ont montré que le principe d'ajustement du profil d'équilibre décrit par P. Bruun (1962) n'a fonctionné que dans la partie orientale de la plage du Vougot, plus à l'ouest, l'action des transits longitudinaux a perturbé les transferts transversaux entre la dune et la plage; pour la plage de Boutrouilles, la présence du Stabiplage ${ }^{\circledR}$ a renforcé les circulations de retour qui ont exporté le matériel érodé à la dune en limite aval de plage intertidale et/ou des petits fonds. Le suivi pluriannuel de ces deux cordons dunaires a montré que l'épisode du 10 mars 2008 était exceptionnel du point de vue du bilan sédimentaire, les volumes érodés ont atteint entre -5 et $-12 \mathrm{~m}^{3} / \mathrm{m}$.l. Il a aussi permis de voir que l'ensemble des processus qui agissent sur les échanges sédimentaires entre la plage et la dune, contrôle en partie l'action érosive des tempêtes. Les hivers marqués par une fréquence plus importante d'épisodes tempétueux combinés à une forte marée, ne permettent pas le réengraissement efficace du système dunaire qui devient alors plus vulnérable face aux tempêtes printanières.

$$
\text { MOTS CLÉ : dune - tempête - érosion - bilan sédimentaire - runup }
$$

\section{ABSTRACT}

Impact of storms on dune erosion: lessons from the $10^{\text {th }}$ March 2008 storm Analysis of dune erosion during the $10^{\text {th }}$ March 2008 storm has been carried out on two sites located on the north Finistère: the dune of Vougot beach (Guissény district) corresponding to a "natural" massive dune complex, and the dune of Boutrouilles beach (Kerlouan district) forming a narrower foredune, protected in its toe by a geotextile tube structure, hydraulically filled with dredged sands, named Stabiplage ${ }^{\circledR}$. For both sites of Vougot and Boutrouilles, the storm generated a maximum retreat reaching respectively 6 and $3 M$. This erosion is mainly due to the combination of strong waves and high spring tide level (coeff. 
106) which has generated extreme water levels exceeding 1 to 1.80 m over the altitude of the dune toe. Beach/dune profile measurements have showed that the adjustment of equilibrium profile, which was assumed and described by P. Brumn (1962) has well operated on the eastern part of the Vougot beach; further to the west longshore sediment transport has interfered cross-shore transfers between dune and beach. Concerning Boutrouilles beach, the existence of Stabiplage ${ }^{\circledR}$ has reinforced rip currents, which had exported sand eroded from the dune towards the low limit of intertidal beach and/and shallow water. The pluriannual survey of both dunes has indicated that the $10^{\text {th }}$ March event was exceptional in terms of sedimentary budget: volumes eroded reached between -5 and $-12 \mathrm{~m}^{3} / \mathrm{l} . \mathrm{m}$. The survey has also allowed to see that processes occurring on sedimentary transfers between dune and beach, control partially storm erosion. Winters characterized by high frequency of storms combined with high spring tide, do not allow efficient regeneration of the dune system, which then becomes more vulnerable to spring storms.

KEY WORDS : dune - storm - erosion - sediment budget - runup

L'analyse et la compréhension des processus d'érosion des dunes lors des tempêtes reste un élément déterminant car le cordon dunaire représente souvent le dernier rempart protégeant de la submersion les zones basses situées en arrière (van de Graaf, 1977; Stauble et al., 1990; Louisse et van der Meulen, 1991 ; Erchinger, 1992 ; Ruessink et Jeuken, 2002 ; Gómez-Pina et al., 2002). Dès la fin des années 1960, l'érosion des dunes par les tempêtes a fait l'objet d'un certain nombre d'études ${ }^{1}$ reposant sur des approches avant tout théoriques ou expérimentales (Edelman, 1968, 1972; van der Meulen et Gourlay, 1968). Elles montrèrent le rôle respectif des différents paramètres intervenant dans les processus d'érosion tels que la morphologie (hauteur et pente de l'escarpement de dune), la sédimentologie (granulométrie et cohésion des sables), ou l'hydrodynamisme (conditions de marée et de houle) (van de Graaff, 1977; Vellinga, 1982, 1984 ; Kriebel et Dean, 1985, 1986; Carter et Stone, 1989, Carter et al., 1990; Pye et Neal, 1994). Dans une étude méthodologique basée sur une approche probabiliste, J. van de Graaff (1986) démontra que le paramètre "submersion ", déterminé par le niveau d'eau à la côte combinant la marée astronomique et la surcote, était le premier facteur intervenant dans les processus d'érosion des dunes (82,8\% de variance). La taille des grains de sables était le second facteur prépondérant $(7,3 \%)$, suivi de la durée de submersion $(2,6 \%)$ et de la forme du profil initial $(1,3 \%)$, alors que la hauteur de la houle n'intervenait que de façon mineure $(0,9 \%$ de variance). Le rôle respectif par ordre d'importance de ces différents paramètres dans les processus d'érosion des dunes a par ailleurs été confirmé dans une étude récente (den Heijer et al., 2008).

Ces travaux débouchèrent sur le développement de modèles numériques simulant le recul des dunes généré par les tempêtes. La première approche repose sur le principe d'ajustement du profil d'équilibre décrit par P. Bruun (1962) qui stipule que le volume érodé à la dune vient engraisser la plage, assurant ainsi une conservation du bilan sédimentaire sur l'ensemble du profil transversal (Edelman, 1972; van de Graaff, 1977; Vellinga, 1982, 1984; Kriebel et Dean, 1985, 1986). L'importance du recul de la dune est alors fonction de l'élévation du plan d'eau au moment de la tempête, qui elle-même détermine l'allure du profil du haut de plage post-tempête suivant la relation : $z=0,415(x+4,5)^{0,5}-0,88$; où $z$ est la profondeur (en $\mathrm{m}$ ) sous le niveau d'eau extrême atteint lors de la tempête, et $x$ la distance (en $\mathrm{m}$ ) à partir de l'intersection entre le profil posttempête et le niveau d'eau extrême. La limite des transferts sédimentaires vers le bas de plage est fonction de la relation : $h_{\mathrm{b}}=1,28 H_{\mathrm{b}}$ (fig. 1 ).

1. Ces premiers travaux ont été réalisés par le laboratoire néerlandais Delft Hydraulics en relation avec le Rijkswaterstaat (département des transports et des travaux publics hollandais). 
Figure 1 : Modèle d'érosion des dunes simplifié (d'après J. van de Graaff, 1977, modifié)

Simple dune erosion model (after J. van de Graaff, 1977, modified)

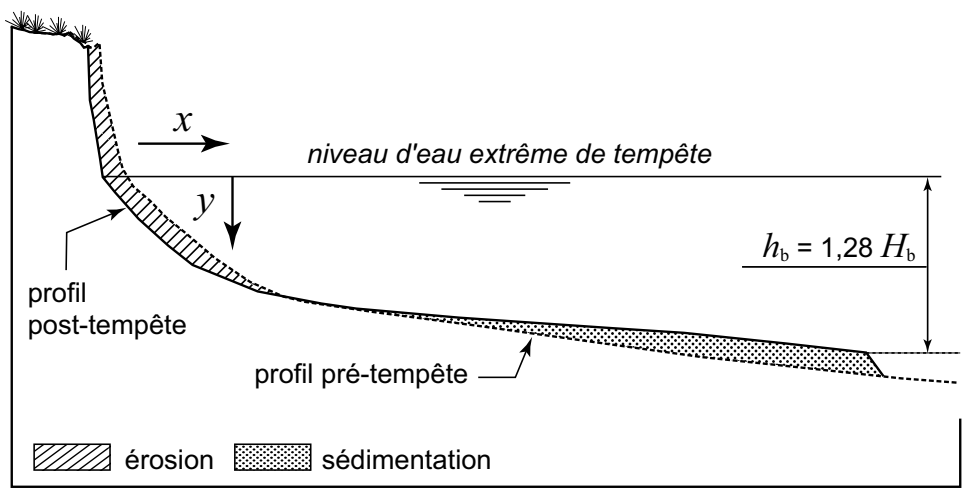

S. A. Hugues et T.-Y. Chui, (1981, in Carter et al., 1990) ont montré que plus de $70 \%$ du volume sédimentaire érodé à la dune intervient avant que les plus hauts niveaux d'eau ne soient atteints. C'est principalement l'attaque des premières vagues qui érode le matériel de la dune entraînant l'exhaussement de la plage, de telle sorte que l'épaisseur de la tranche d'eau submergeante reste constante. Très vite, un nouvel équilibre s'instaure alors entre le profil de plage « de tempête » et les conditions hydrodynamiques tempétueuses, diminuant ainsi l'action érosive des vagues.

Une seconde approche modélisatrice reposant sur l'analyse des processus dynamiques a été proposée en tenant compte de l'impact du jet de rive sur le front de dune (Fisher et Overton, 1984; Larson et al., 2004; Erikson et al., 2007). Ainsi, le recul total de la dune, et par là même le volume de sable érodé, est fonction de la fréquence et de l'intensité de chaque jet de rive dès lors que la hauteur de ce dernier (runup) est supérieure à celle du pied de dune (Overton et Fisher, 1988). Ces processus sont à l'origine du sapement de pied de dune qui, à terme, entraîne une déstabilisation de l'escarpement facilement identifiable par la présence de formes d'érosion telles que les fissures de tension, les éboulements, ou les glissements (Carter et Stone, 1989; Carter et al., 1990; Erikson et al., 2007). De nombreuses études ont toutefois insisté sur le rôle déterminant de la végétation racinaire qui permet de maintenir une forte pente de l'escarpement $\left(40^{\circ}\right.$ à $43^{\circ}$ ) après érosion, alors que pour les dunes non végétalisées, les phénomènes de glissement et d'éboulement qui affectent le versant érodé entraînent une diminution de la pente $\left(32^{\circ}\right.$ à $\left.34^{\circ}\right)$ (Carter, 1980). C'est sur ce principe que P. Ruggiero et al. (2001) ont proposé un modèle visant à évaluer la sensibilité des cordons dunaires à l'érosion générée par le déferlement des vagues de tempête. La démarche consiste à mettre en relation la hauteur des niveaux d'eau extrêmes à la côte, définie par la somme du runup et de la marée observée, avec celle du pied de dune; lorsque la tranche d'eau est supérieure à l'altitude du pied de dune l'érosion intervient. Cette approche méthodologique a permis entre autre d'évaluer la vulnérabilité des îles barrières soumises à l'action des ouragans le long de la côte est des Etats-Unis (Sallenger, 2000; Salenger et al., 2002; Stockdon et Thompson, 2007; Stockdon et al., 2007).

L'objectif de cet article a été d'analyser l'impact morphogénique de la tempête du 10 mars 2008 (cf. Cariolet et al., ce volume) sur les systèmes dunaires de deux plages du Finistère nord : la plage du Vougot à Guissény et de Boutrouilles à Kerlouan (fig. 2) et, à partir de là, d'étendre la réflexion à la problématique générale de l'action des tempêtes sur l'érosion des dunes. Les effets de cette tempête sur ces deux sites ont été d'autant mieux enregistrés que cet épisode est intervenu alors que ces deux plages faisaient l'objet d'un suivi topo-morphologique à haute fréquence depuis plusieurs années. Dans un premier temps, nous avons cherché à voir si les processus observés du point de vue morphosédimentaire et hydrodynamique répondaient aux modèles théoriques présentés ci-dessus. Dans un second temps, l'analyse a été poursuivie en replaçant cet évènement au sein de l'évolution pluriannuelle mesurée depuis l'année 2004 pour la plage du Vougot, et 2006 pour la plage de Boutrouilles; l'objectif étant d'étudier le rôle de ces épisodes morphogènes dans 
Serge Suanez, Jean-Marie Cariolet

le fonctionnement à plus long terme des dunes. Enfin, nous avons cherché à déterminer les principaux paramètres intervenant dans le processus d'érosion; dans le même temps le travail a porté sur l'analyse de la résilience des ces cordons.

\section{Contexte géographique}

Les deux sites d'étude se situent sur la côte nord du Finistère, ils correspondent à la plage du Vougot située sur la commune de Guissény et à la plage de Boutrouilles sur la commune de Kerlouan, et sont localisés de part et d'autre de la pointe de Neiz Vran (fig. 2). L'ensemble de cette zone littorale qui s'étend de Landéda-Plouguerneau à l'ouest à la baie de Goulven à l'est, est constitué d'une large plateforme à écueils s'étendant en contrebas du plateau du Léon. Le contact entre la plate-forme littorale et la partie continentale du plateau se fait par un escarpement en partie d'origine tectonique, de 30 à $50 \mathrm{~m}$ de commandement suivant les secteurs. À l'ouest, dans le secteur de Plouguerneau, cet escarpement est battu par la mer; en se déplaçant vers l'est jusqu'à la baie de Goulven, cet abrupt prend l'allure d'une falaise morte déconnectée de la mer par des plaines littorales sédimentaires, en partie fermées par des cordons littoraux, édifiés durant la transgression holocène (Hallégouët, 1971; Hallégouët, 1978; Guilcher and Hallégouët, 1991). La morphologie générale de la zone du Vougot et de Boutrouilles illustre très bien ce dernier cas (fig. 2).

Les houles principales arrivant sur l'ensemble du secteur proviennent essentiellement de l'ouestnord-ouest (Suanez et al., 2007). Les hauteurs $\left(\mathrm{H}_{\mathrm{mo}}\right)$ modales sont comprises entre 1 et 1,5 m, les périodes modales $\left(\mathrm{T}_{\text {pic }}\right)$ entre 9 et 10 secondes. Le littoral de Guissény est relativement bien protégé des houles d'ouest à nord-ouest par la plate-forme hérissée d'îlots et de récifs émergeants à basse mer (Golhédoc, Enez Du). Ces derniers rendent la circulation hydrosédimentaire à la côte complexe en provoquant d'importants phénomènes de diffraction à l'origine des nombreuses queues de comètes construites à l'arrière de ces obstacles (fig. 2). Ce sont également eux qui donnent au tracé du cordon dunaire une forme courbe convexe en procurant à la côte un effet d'abri. La plage de Boutrouilles est aussi protégée de la houle d'ouest par la pointe de Neiz Vran, elle reste cependant plus exposée aux houles de nord-ouest. D’après les données du SHOM, le marnage se situe aux alentours de 7,10 m (cette valeur correspond à la moyenne du marnage au niveau de l'Aber Wrac'h : 6,7 m et de Roscoff : 7,55 m).

\section{LE CORdon dunAire de la Plage du Vougot}

Au niveau de la plage du Vougot, le cordon dunaire s'étire sur environ $2 \mathrm{~km}$ depuis la falaise morte du Zorn, suivant une direction sud-ouest nord-est (fig. 3). Il protège une vaste zone poldérisée déconnectée de la mer au niveau de Porz Olier, par la construction d'une digue artificielle en 1834 (Bodéré et al., 1983). D’après A. Guilcher et B. Hallégouët (1991), la partie interne de ce cordon a pu se former à l'âge du Bronze lorsque la mer a atteint son niveau actuel. Un second cordon, externe, s'est mis en place au début de l'âge du Fer ( \pm 2400 ans BP) durant un léger stade régressif du niveau de la mer. Enfin, un stade ultime de progradation dunaire est bien identifié entre le $\mathrm{XVII}^{\mathrm{e}}$ et le XVIII ${ }^{\mathrm{e}}$ siècle, notamment dans le secteur étudié de Plouguerneau à Guissény. Cette dune culmine à près de $13 \mathrm{~m}$ d'altitude NGF, et se présente sous la forme d'un complexe dunaire massif d'une largeur comprise entre $250 \mathrm{~m}$ et $400 \mathrm{~m}$. La plage intertidale marquée quant à elle par une pente moyenne faible $(\tan \beta=0,025)$ est caractérisée par un contexte morphodynamique dissipant (Wright et Short, 1984). Pour ce qui est de la granulométrie, la taille des sables dunaires est comprise entre 200 et $250 \mu \mathrm{m}$, elle tend à augmenter en haut de plage (entre 250 et $315 \mu \mathrm{m})$.

La partie interne du massif dunaire est colonisée par une végétation rase extrêmement dense, constituée essentiellement d'une pelouse aérohaline. Sur la partie externe du cordon dunaire située proche de la mer, on trouve une ceinture de graminées (sp. Ammophila arenaria) dont le système 


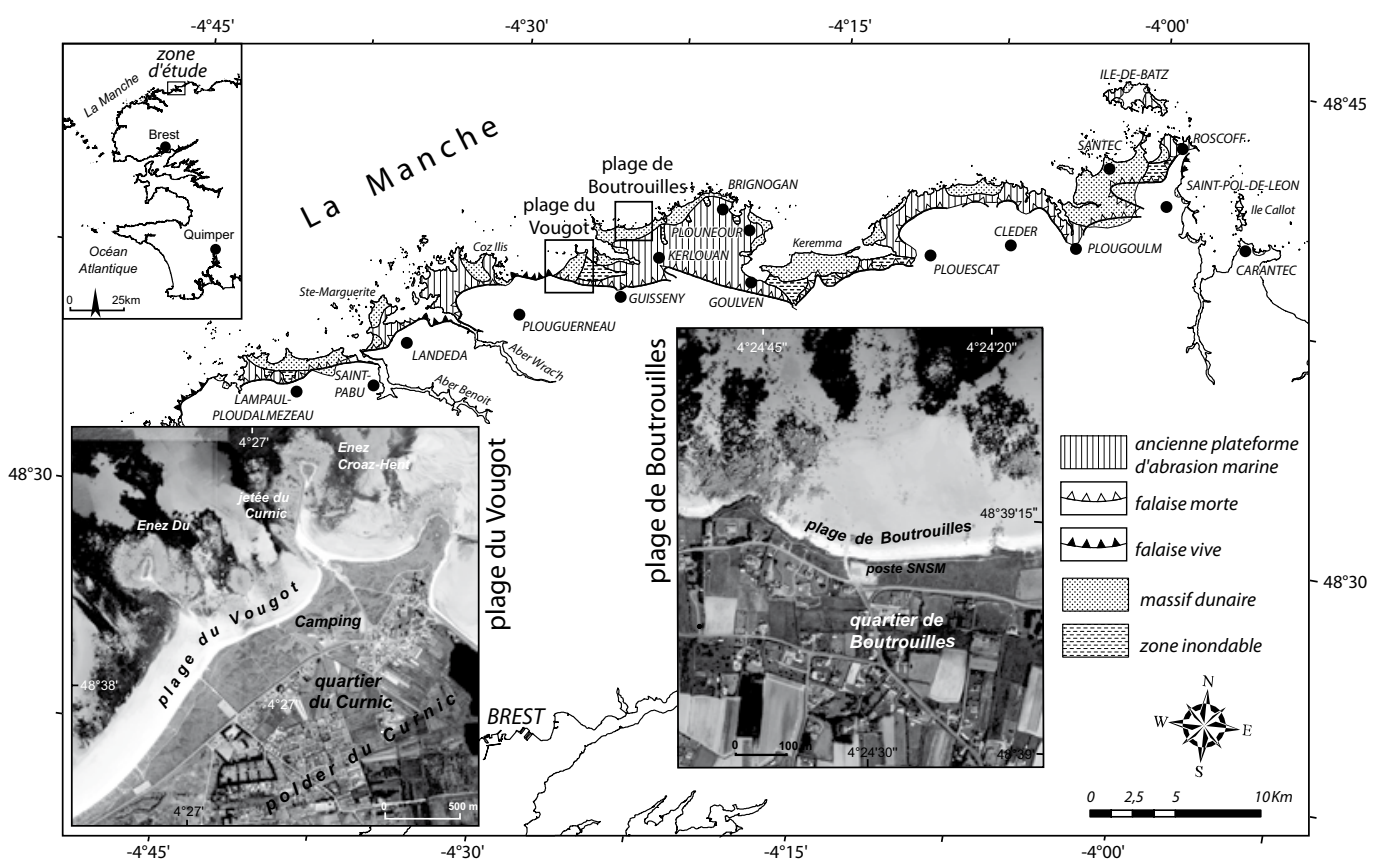

Figure 2 : Localisation des sites d'étude (fond de carte d'après B. Hallégouët, 1978, modifié) Location of the study areas (map after B. Hallégouët, 1978, modified)

racinaire apparaît en coupe au sommet de l'escarpement. Le réseau de racines très dense pénètre à plus de 1,5 m de la surface de la dune, il augmente ainsi la cohésion des sables dans cette partie haute de l'escarpement qui présente une pente subverticale (photo 1A). À certaines périodes de l'année, le versant externe de la dune présente de nombreuses formes d'érosion telles que des falaises, des glissements ou des éboulements qui témoignent de l'attaque fréquente et répétée des tempêtes (photos 1B, 1C et 1D). Cette érosion, concentrée essentiellement sur la partie orientale du cordon dunaire (fig. 3), a fait l'objet de nombreuses études depuis ces dernières années; la vitesse de recul maximum calculée dans ce secteur entre la fin des années 1970 et 2000 atteint $-0,6$ à $-0,75 \mathrm{~m} / \mathrm{an}$, elle s'est toutefois accélérée entre 2004 et 2009 pour atteindre -1,2 à -1,5 m/ an (Sparfel et Suanez, 2007; Suanez et al., 2007; Cariolet et Suanez, 2009, Suanez et al., 2010).

L'analyse du bilan sédimentaire du système plage/dune de ce secteur effectuée sur la période 2005-2009 a montré qu'une grande partie du matériel érodé à la dune venait dans un premier temps engraisser le haut de plage (Suanez et al., 2010). Ce matériel est ensuite repris par des courants de dérive orientés d'est en ouest; cette circulation est liée à la diffraction de la houle autour de l'écueil d'Enez Du (fig. 3). Cette dynamique hydrosédimentaire explique en partie que plus l'on se déplace vers l'ouest (en direction de la falaise du Zorn), plus l'érosion du cordon dunaire diminue jusqu'à devenir inexistante (Sparfel et Suanez, 2007). À l'heure actuelle, on peut donc dire que ce cordon dunaire correspond en grande partie à une forme héritée dont le bilan sédimentaire est globalement déficitaire, surtout dans sa partie orientale. Toutefois, comme on le verra, une alimentation saisonnière centrée sur la période estivale peut engraisser le pied de dune qui alors s'exhausse momentanément. Ces apports d'origine éolienne proviennent de la plage intertidale qui ne cesse de perdre du matériel comme l'ont montré les mesures effectuées entre 2005 et 2009 (Suanez et al., 2010). 

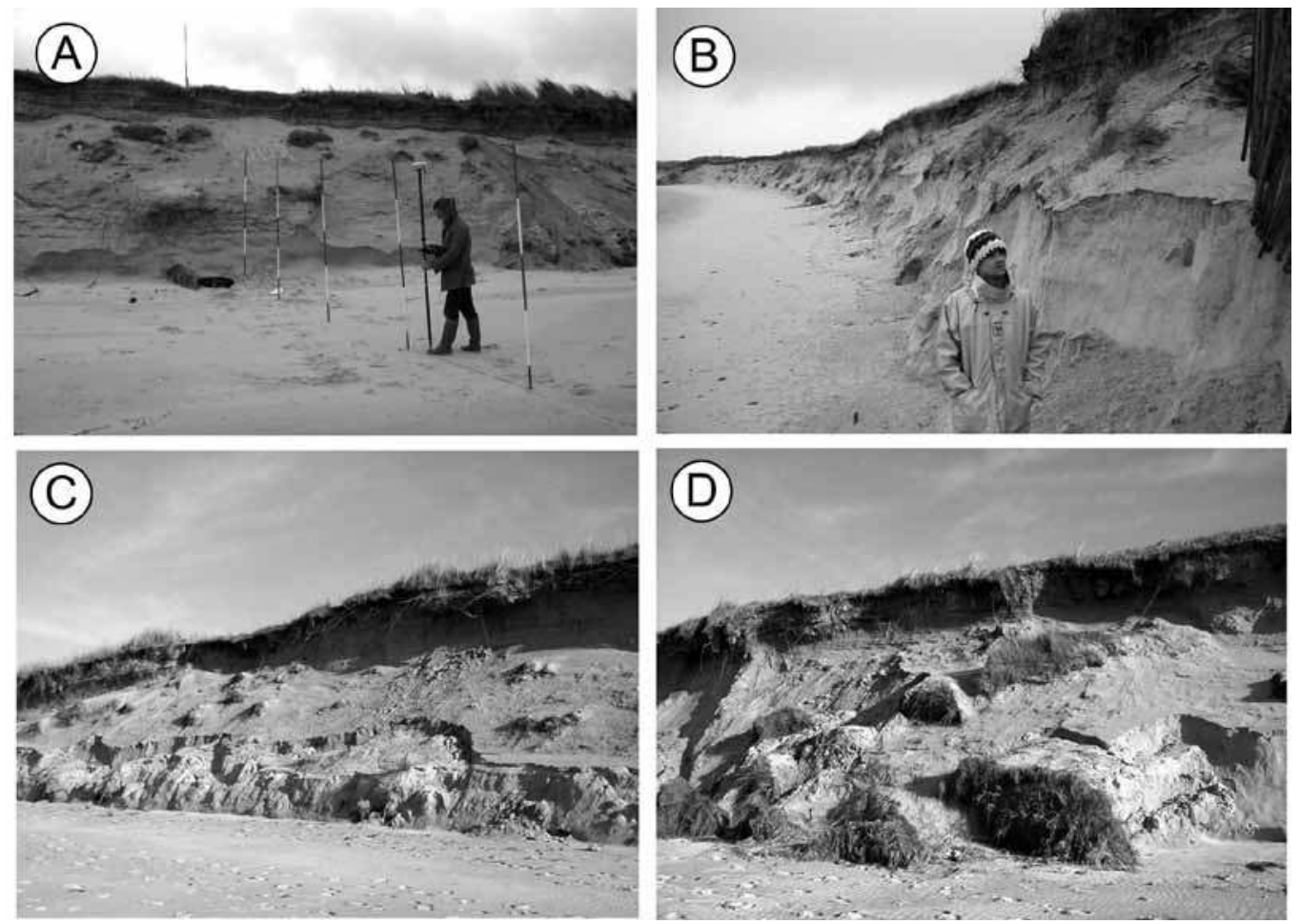

Photo 1 : Cordon dunaire de la plage du Vougot dans sa partie en érosion

A : profondeur du réseau racinaire de la ceinture de graminées (sp. Ammophila arenaria) délimitée par l'horizon sombre en haut d'escarpement. B : falaise d'érosion taillée par une tempête (cliché S. Suanez, 22 mars 2007). C : phénomènes de glissements affectant le versant externe de la dune après une tempête (cliché S. Suanez, 6 mars 2006). D : éboulements affectant le versant externe de la dune après une tempête (cliché S. Suanez, 6 mars 2006 ). Eroded sector of the foredune of Vougot beach

A: depth of vegetation roots penetration (sp. Ammophila arenaria) delimited by the dark layer on the top of dune scarp. B: undercutting during storm leading to vertical scarp. C: slumps affecting dune slope profile after storm. D: failures affecting dune slope profile after storm.

\section{LE CORDON DUNAIRE DE LA PLAGE DE Boutrouilles}

Le site de Boutrouilles présente un cadre géographique assez similaire à celui du Vougot. Le cordon dunaire dont la période de construction est probablement similaire à celle de la dune du Vougot $^{2}$ correspond à une forme héritée. Il s'étire sur environ 300 m suivant une direction ouest est, et barre une zone basse située en arrière, en partie urbanisée, et dont l'altitude est proche ou en dessous du niveau marin. À la différence de la plage du Vougot, le cordon dunaire est moins élevé et moins large, il culmine à $11 \mathrm{~m}$ d'altitude NGF pour une largeur comprise entre 50 et $70 \mathrm{~m}$ de largeur (fig. 3). La taille du grain moyen des sables dunaires est similaire à celle de la dune du Vougot (entre 250 et $200 \mu \mathrm{m}$ ), à l'inverse, le matériel du haut de plage ayant été complètement remanié lors des travaux est un peu plus grossier (entre 315 et $400 \mu \mathrm{m}$ ).

2. Aucune étude chrono-stratigraphique n'a été réalisée sur ce secteur. Toutefois, la proximité des deux sites, et la similitude du contexte morphologique laissent à penser que les phases de construction dunaire ont été les mêmes sur les plages du Vougot et de Boutrouilles. 


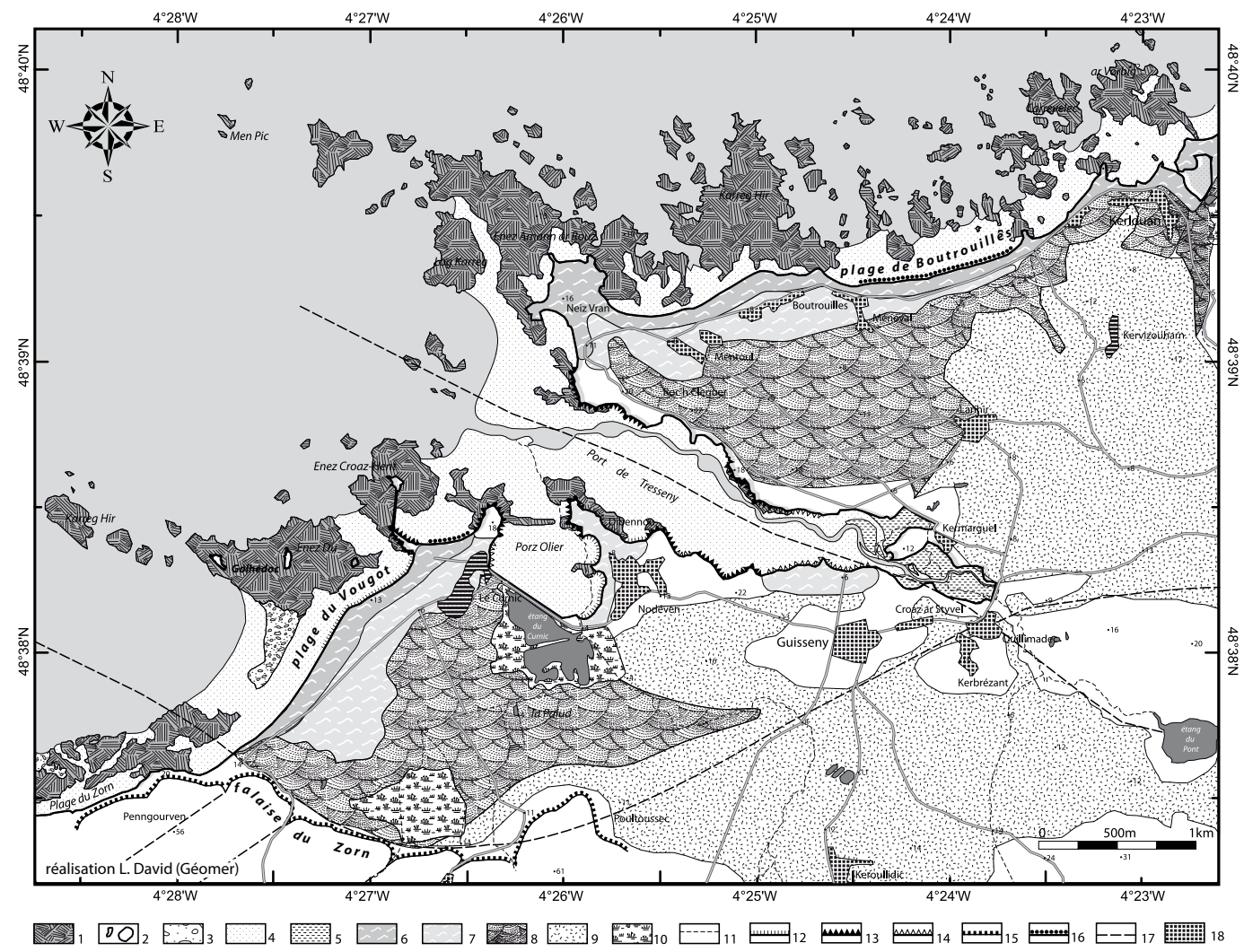

Figure 3 : Carton géomorphologique de la zone d'étude (d'après B. Hallégouët, 1971)

1 : platier. 2 : îlot. 3 : épandage de galets. 4 : sable de plage. 5 : vasière. 6 : dune externe. $7:$ dune interne. 8 : formation sablo-limoneuse. 9 : limons. 10 : marais. 11 : cours d'eau. 12 : falaise d'érosion dunaire. $13:$ falaise vive rocheuse. 14 : falaise vive de head. 15 : falaise morte. 16 : ouvrage de défense côtière. 17 : faille. 18 : zone urbanisée. Geomorphological map of the study area (after B. Hallégouët, 1971)

1: wave-cut platform. 2: island. 3: gravel sheet. 4: sand beach. 5: mud flat. 6: outer dune. 7: inner dune. 8: sandy-silt deposit. 9: silt. 10: brackish marsh. 11: river. 12: dune scarp. 13: rocky cliff. 14: periglacial cliff. 15: abandoned cliff. 16: sea defence structure. 17: fault. 18: urban zone.

Comme pour la dune du Vougot, ce cordon dunaire, soumis pendant des décennies à l'action érosive de la mer (photo 2A), recule en moyenne de - $0,15 \mathrm{~m} /$ an depuis 1952 ; ce recul s'est toutefois accéléré sur la période récente (de 2000 à 2005) pour atteindre -0,7 m/an (Cariolet et Suanez, 2009). Dans le but de stopper cette érosion la décision de protéger la dune a été prise par les élus locaux. Entre les mois d'octobre et de novembre 2006, un ouvrage longitudinal de type Stabiplage ${ }^{\circledR}$ a été mis en place au pied du cordon dunaire sur une longueur de 270 mètres linéaires afin de fixer le trait de côte (photos 2B, 2C). Il s'agit d'une structure en matériau géocomposite formant une enveloppe perméable qui, une fois injectée de sédiments, constitue un corps monolithique de section elliptique (Cariolet et al., 2008). Dans le même temps, le versant externe a été reprofilé et une ceinture d'oyat a été plantée le 7 mars 2007 en son sommet afin de renforcer le maintien des sables dans cette partie haute du profil. Quelques jours plus tard, le 23 mars 2007, une tempête associée à une pleine mer de vive-eau a sapé le pied de dune entraînant des phénomènes de glissement qui ont emporté les graminées (Cariolet et al., 2008). Au mois de mai 2007, le versant externe de la dune a été de nouveau reprofilé et une nouvelle ceinture d'oyat a été plantée (photo 2D). 

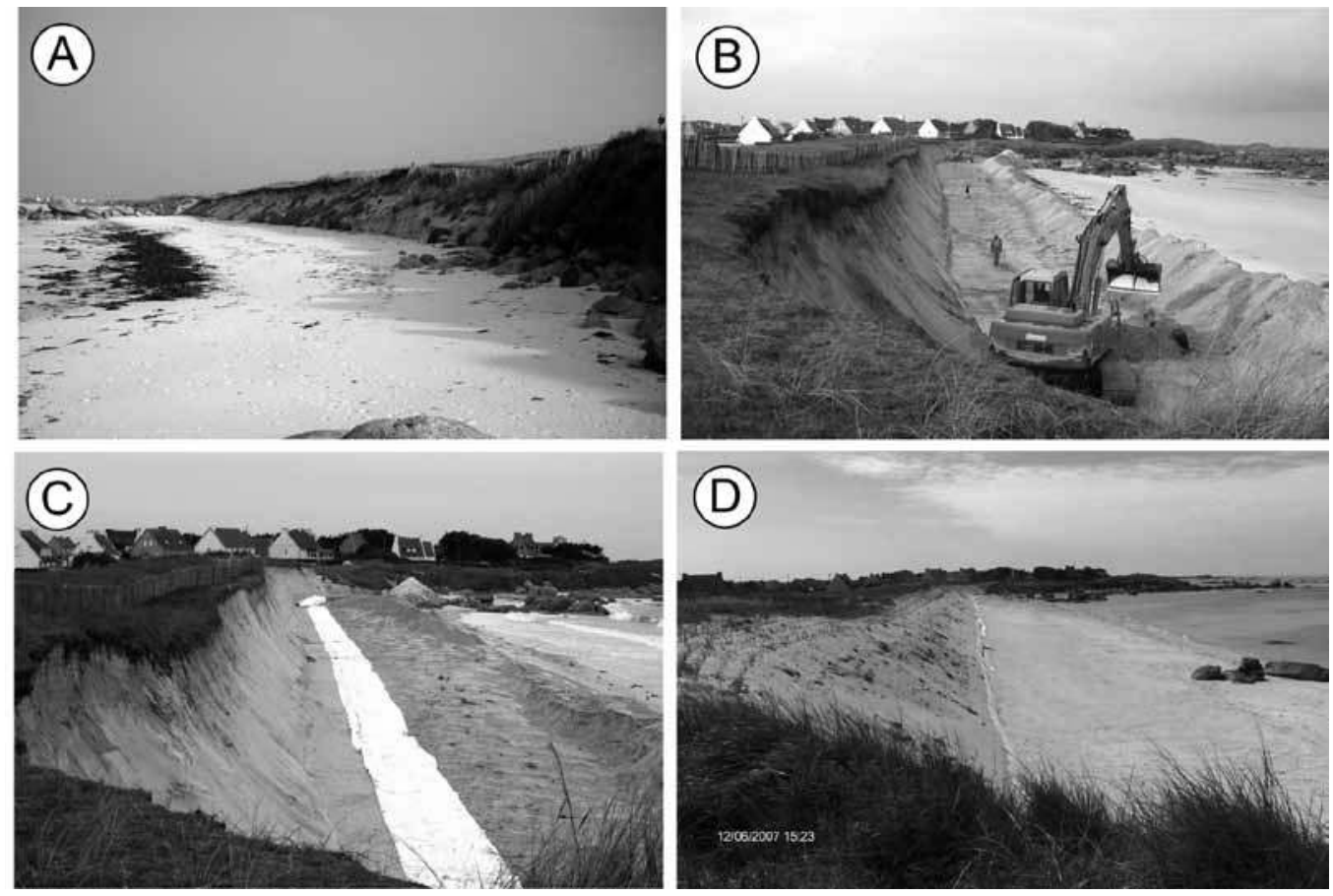

Photo 2 : Cordon dunaire de la plage de Boutrouilles.

A : versant externe de la dune avant les travaux de protection. On distingue des formes d'érosion telles que des falaises et des éboulements. B : décaissement du pied de dune afin de poser l'ancrage du Stabiplage ${ }^{\circledR}$ (cliché A. Démon, 19 octobre 2006). C : pose de la structure Stabiplage ${ }^{\circledR}$ avant injection (cliché S. Suanez, 24 octobre 2006). D : versant externe de la dune reprofilé et planté d'oyat dans sa partie sommitale (cliché Espace Pur, 12 juin 2007 ). Foredune of Boutrouilles beach

A: extern dune slope before works of dune protection. We distinguish erosion forms such as dune scarp and failures. B: digging of dune toe in a way to install Stabiplage ${ }^{\circledR}$ cramping. C: installation of Stabiplage ${ }^{\circledR}$ structure before sediment injection. D: extern dune slope reshaped and stabilized by Ammophila plantation in its upper part.

Les dynamiques hydrosédimentaires observées au sein de cette cellule littorale sont largement commandées par des transferts transversaux (Cariolet et al., 2008). Ce fonctionnement s'explique par le fait que la plage de Boutrouilles est fermée à ses deux extrémités orientale et occidentale par des «saillants » rocheux qui limitent considérablement les transits longitudinaux (fig. 3). À cela s'ajoutent les effets réfléchissants induits par la présence du Stabiplage ${ }^{\circledR}$ qui génèrent aussi des déplacements sédimentaires transversaux en haut de plage (Cariolet et al., 2008).

\section{Méthodes et données utilisées}

\section{MÉTHODE DE LEVÉ ET DE TRAITEMENT DES DONNÉES TOPO-MORPHOLOGIQUES}

Les données topo-morphologiques acquises dans le cadre de cette étude reposent sur des levés de profils de plage intégrant la dune et le haut de plage intertidale ${ }^{3}$ : trois profils de plage au Vougot et deux à Boutrouilles (fig. 4). Ces mesures, effectuées à une fréquence mensuelle, ont commencé

3. Dans certains cas, cette limite correspond au contact entre le sable et le platier rocheux (cf. P2 et P3 sur la plage du Vougot). Pour les autres profils (Pl sur la plage du Vougot et les 2 profils de Boutrouilles), elle correspond grossièrement au niveau de mi-marée (niveau moyen de la mer) 
au mois de juillet 2004 et sont toujours en cours pour la plage du Vougot. À Boutrouilles, le suivi topo-morphologique a été réalisé avec la même fréquence entre les mois de novembre 2006 et septembre 2008. À ces levés s'ajoute un relevé du front de dune de la plage du Vougot qui est réalisé tous les ans. Ces mesures sont effectuées au DGPS en temps réel, et ont été raccordées au système géodésique français à partir d'une borne IGN ${ }^{4}$ située sur la presqu'île de Neiz Van (fig. 4). La mise en place de points de calage qui sont systématiquement relevés à chaque campagne de mesure permet d'estimer une marge d'erreur inhérente à la mesure comprise entre $+/-5 \mathrm{~cm}$ en $x$ et $\mathrm{y}$, et $<+/-1,5 \mathrm{~cm}$ en $z$. Les bilans sédimentaires ont été calculés à partir de la méthode des surfaces verticales. Deux sections du profil de plage/dune ont été retenues pour le calcul des bilans sédimentaires : (i) la dune stricto sensu en prenant pour limite aval le pied de dune pour la plage du Vougot (comprise entre 15 et $18 \mathrm{~m}$ par rapport à la tête de profil), et le pied du Stabiplage ${ }^{\circledR}$ pour la plage de Boutrouilles (se situant pour les deux radiales à $10 \mathrm{~m}$ par rapport à la tête de profil); (ii) la haute plage intertidale jusqu'à une distance aval variant suivant les radiales de 150 à $210 \mathrm{~m}$ par rapport à la tête de profil.

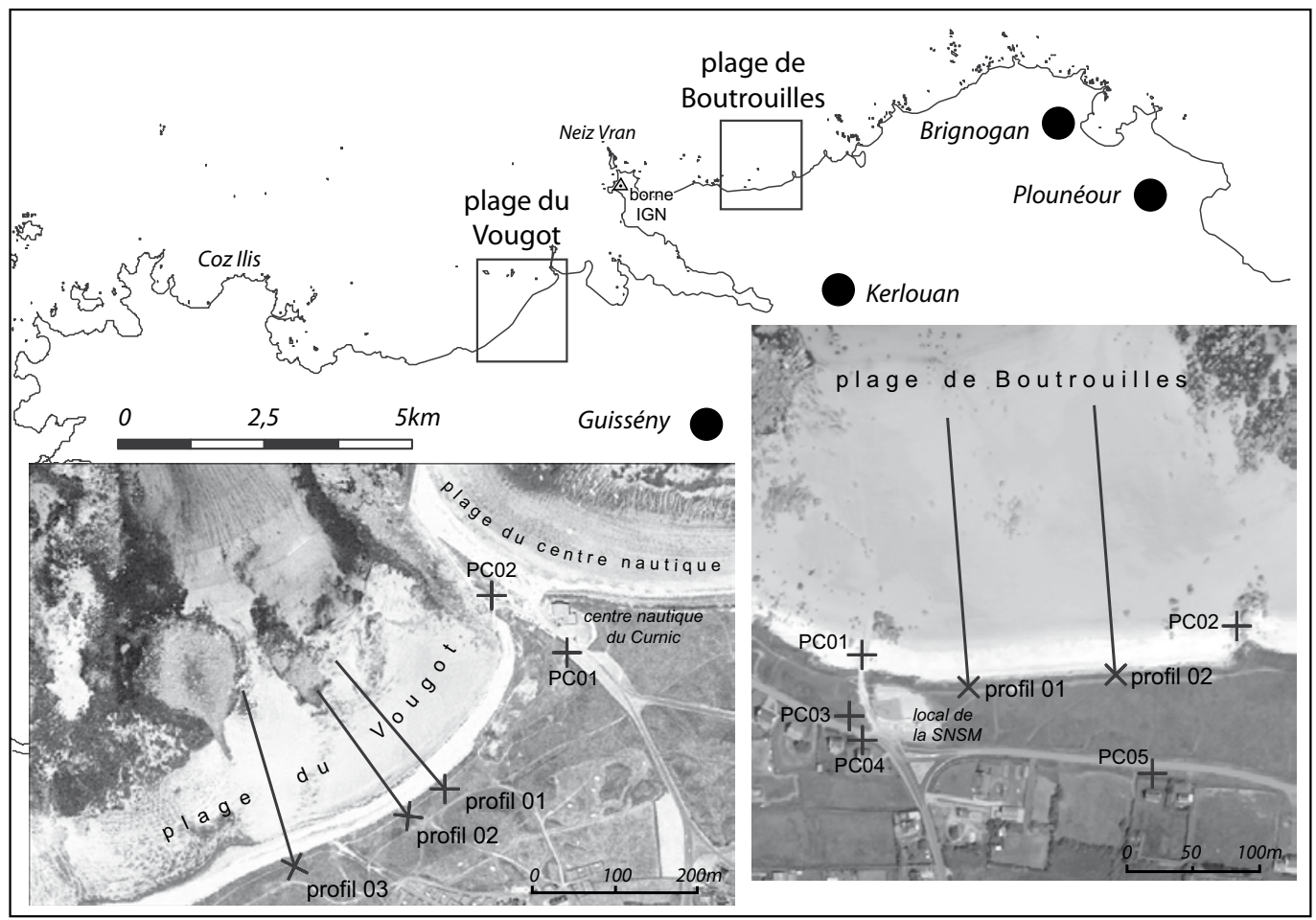

Figure 4 : Localisation des profils de plage et des points de calage (PC) mis en place pour la mesure de terrain sur les plages du Vougot et de Boutrouilles

Boutrouilles beaches

Location of beach profiles and control points (CP) established for field measurements on Vougot and

4. Borne IGN Kerlouan I. N site : 2909101 - point A. Borne en granit gravée IGN. Coordonnées système NTF - projection Lambert I nord, est : 101800,25 m; nord : 128274,81 m; altitude NGF (IGN69) : 16,38 m. 
Serge Suanez, Jean-Marie Cariolet

\section{ACQUiSITION ET DE TRAITEMENT DES DONNÉES MÉTÉOMARINES}

L'analyse des conditions météo-atmosphériques (vent, pression barométrique) relatives à la tempête du 10 mars 2008 a été réalisée à partir de données acquises auprès de la station météorologique du réseau Météo France située à Brignogan (fig. 4). L'étude des conditions hydrodynamiques a reposé quant à elle sur le calcul des niveaux d'eau extrêmes à la côte. Cette information a été obtenue en sommant les deux paramètres agissant sur la déformation du plan d'eau : la marée observée (marée astronomique + surcote) et le rumup (effets combinés du setup et du jet de rive générés par la houle). La marée observée provient des enregistrements du marégraphe de Roscoff situés à une trentaine de kilomètres de la zone d'étude (fig. 2); les données de houle au large utilisées pour le calcul du runup ont quant à elles été obtenues par modélisation auprès du LNHE-EDF et du SHOM. Le calcul du runup s'est fait à partir des équations de H. F. Stockdon et al. (2006).

$R_{2}=1,1\left(0,35 \beta_{f}\left(H_{0} L_{0}\right)^{1 / 2}+\frac{\left[H_{0} L_{0}\left(0,563 \beta_{f}^{2}+0,004\right)\right]^{1 / 2}}{2}\right)$

$R_{2}=0,73 \beta_{f}\left(H_{0} L_{0}\right)^{1 / 2}$

où, $R_{2}$ : hauteur atteinte par $2 \%$ des plus hauts jets de rive, $\beta_{f}$ : pente de la plage, $H_{0}$ et $L_{0}$ : hauteur et longueur d'onde de la houle au large; la formule (1) a été utilisée lorsque $\xi_{0}<0,3$; la formule (2) lorsque $0,3<\xi_{0}<1,25$; où $\xi_{0}$ : nombre d'Iribarren (Battjes, 1974).

Il convient de préciser que les niveaux d'eau extrêmes calculés à partir de cette approche théorique ne correspondent qu'à des ordres de grandeur dont il est difficile d'estimer l'incertitude. En effet, les équations qui ont été utilisées tiennent compte de la houle au large, or, comme nous l'avons montré, la plateforme à écueils induit des phénomènes de diffraction et/ou de réfraction qui modifient considérablement les caractéristiques de houle à la côte. De même, les valeurs de marée enregistrées à Roscoff sont certainement différentes de celles observées sur les plages du Vougot et de Boutrouilles compte tenu de la distance qui les sépare. Toutefois, comme nous le verrons ultérieurement, les hauteurs de niveaux d'eau extrêmes obtenues par cette méthode (que nous pouvons considérer comme «indicatives»), s'accordent avec les observations faites sur le terrain lors de la tempête du 10 mars 2008.

\section{Impacts morphosédimentaires de la tempête du 10 mars 2008}

Comme le montrent les photos comparatives (photo 3), les niveaux d'eau extrêmes à la côte durant la tempête du 10 mars ont été particulièrement hauts sur les deux sites. Cela s'est traduit par une attaque directe du pied et/ou de l'escarpement de dune par les vagues déferlantes, même sur la plage de Boutrouilles, malgré la présence de l'ouvrage de défense Stabiplage ${ }^{\circledR}$. D'un point de vue morphosédimentaire, l'érosion des cordons dunaires a été très importante sur les deux sites. Les mesures effectuées deux jours après l'épisode ont montré que le recul maximum du front de dune de la plage du Vougot avait atteint pratiquement $6 \mathrm{~m}$ (fig. 5). Comme le montre la figure 4, l'érosion croissante d'est en ouest est passée de $-1 \mathrm{~m}$ au niveau des profils $\mathrm{P} 01$ à P06, à plus de -5 m entre les profils P12 et P19. Pour la plage de Boutrouilles, nous ne disposons pas de mesures du front de dune; ceci étant, les observations de terrain réalisées deux jours après la tempête, ont montré que recul de la dune avait été environ $3 \mathrm{~m}$ sur l'ensemble de la plage (photo 3D). 

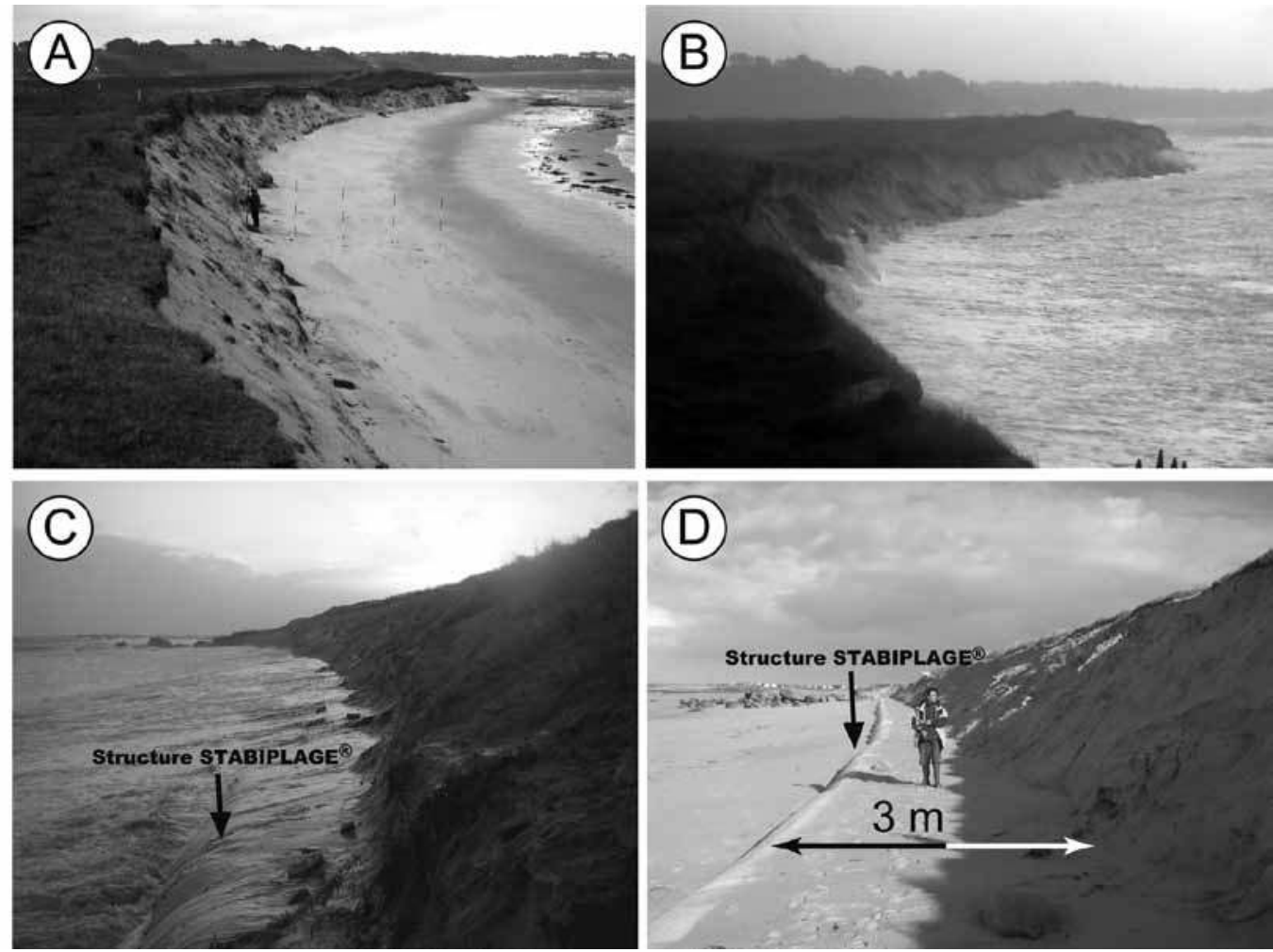

Photo 3 : Attaque du cordon dunaire par les hauts niveaux d'eau durant la tempête du 10 mars 2008

(A) et (B) : cordon dunaire de la plage du Vougot en période d'accalmie et au soir du 10 mars à 19h30. (C) et (D) : cordon dunaire de la plage de Boutrouilles au matin du 10 mars 2008 à 7 h 00, et le 12 mars 2008. Clichés S. Suanez et J.-M. Cariolet.

Wave attack on the dune due to the high water level during the $10^{\text {th }}$ of March 2008 storm (A) and (B): foredune of Vougot beach during calm period and the $10^{\text {th }}$ of March at 7 h 30 PM. (C) and (D): foredune of Boutrouilles beach the $10^{\text {th }}$ of March at $7 \mathrm{hOO} \mathrm{AM,} \mathrm{and} \mathrm{the} 12^{\text {th }}$ of March.

Les mesures de profils de plage/dune effectués sur les deux sites vont dans le même sens (fig. 6). Les trois profils de la plage du Vougot ont enregistré un recul significatif de la dune. Le calcul des volumes sédimentaires effectué sur deux sections du profil de plage (la dune et la haute plage intertidale) indique que pour les profils 1 et 2, la quantité de matériel perdu par le recul de la dune (respectivement $-9,2$ et $-8,51 \mathrm{~m}^{3} / \mathrm{m} . \mathrm{l}$ ) est compensée par un gain sédimentaire du haut de plage (respectivement $+10,24$ et $+16,45 \mathrm{~m}^{3} / \mathrm{m} . \mathrm{l}$ ) (fig. 6A et 6B). À l'inverse, les résultats obtenus pour le profil 3 de la plage du Vougot (fig. 6C), et pour les deux profils de la plage de Boutrouilles (fig. $6 \mathrm{D}$ et $6 \mathrm{E}$ ), indiquent que l'ensemble du système dune/haut de plage est en érosion. Il semblerait toutefois que pour la plage de Boutrouilles des phénomènes d'accrétion, non pris en compte par la mesure, interviennent au-delà d'une profondeur comprise entre -1 et -2 m NGF (fig. 6D et 6E).

\section{Niveaux d'eau extrêmes atteints lors de la tempête du 10 mars 2008}

Comme nous l'avons rappelé en introduction, l'érosion des cordons dunaires intervient dès lors que les niveaux d'eau extrêmes à la côte sont supérieurs à l'altitude du pied de dune ; c'est le principe du Property Erosion Model défini par P. Ruggiero et al. (2001). Cette approche a été utilisée par de nombreux auteurs afin d'expliquer la dynamique de recul des cordons dunaires et/ou de 


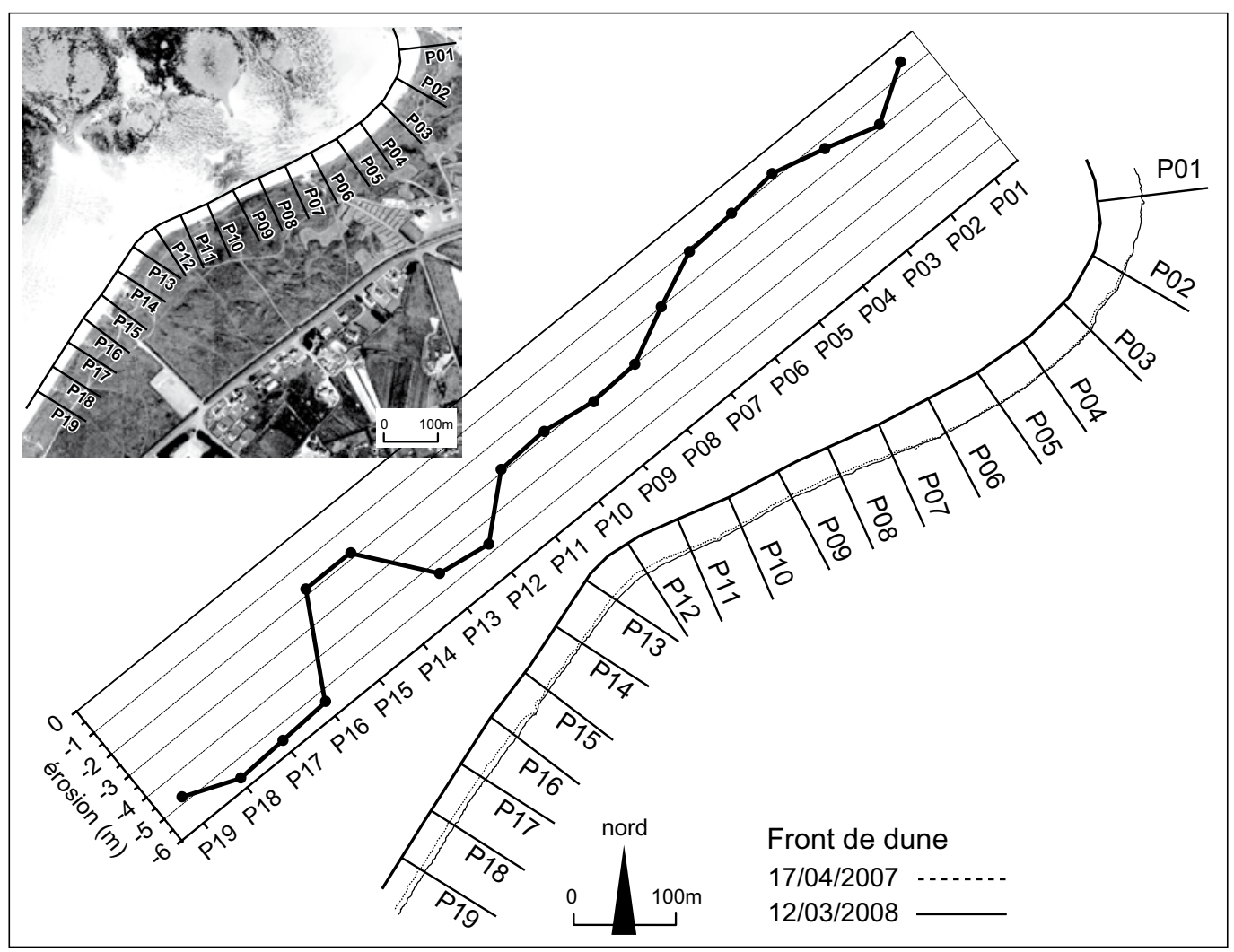

Figure 5 : Recul du front de dune de la plage du Vougot mesuré deux jours après la tempête du 10 mars 2008 (aucune donnée sur la position du front de dune en 2007 n'était disponible au-delà du profil P19)

Foredune retreat of Vougot beach obtained by field measurements two days after the $10^{\text {th }}$ of March storm (no data concerning the 2007's position of the front of the dune was available further profile P19)

définir des modèles de vulnérabilité des cordons littoraux face aux tempêtes (Sallenger, 2000 ; Sallenger et al., 2002; Suanez et Stéphan, 2006; Stockdon et al., 2007 ; Stockdon et Thompson, 2007).

Dans le cadre de cette étude, les résultats montrent que pour les deux sites, les niveaux d'eau extrêmes à la côte atteignant 7,40 m NGF, ont été bien supérieurs à l'altitude du pied de dune quel que soit le profil de plage. Pour la plage de Boutrouilles, les plus hauts jets de rive ont même franchi la hauteur maximale du Stabiplage ${ }^{\circledR}$ (photo 3C) entraînant une érosion de la dune en arrière de l'ouvrage (photo 3D). Dans les deux cas, les niveaux d'eau attaquant le versant externe de la dune ont dépassé d'environ $1 \mathrm{~m}$ à 1,80 m le pied de dune. Les valeurs obtenues ne donnent toutefois que des ordres de grandeur car si l'on tient compte des effets locaux, liés notamment à la force du vent $(>22 \mathrm{~m} / \mathrm{s})$ associée à une très faible pression $(975 \mathrm{hpa})$ (fig. $7 \mathrm{~B}$ et $7 \mathrm{C})$, et qui ne sont pas toujours enregistrés par les marégraphes situés le plus souvent en zone portuaire abritée (Benavente et al., 2006), ces niveaux d'eau ont pu être bien supérieurs. En effet, les observations de terrain durant la pleine mer du soir nous ont montré que certains jets de rive dépassaient la crête de la dune. 

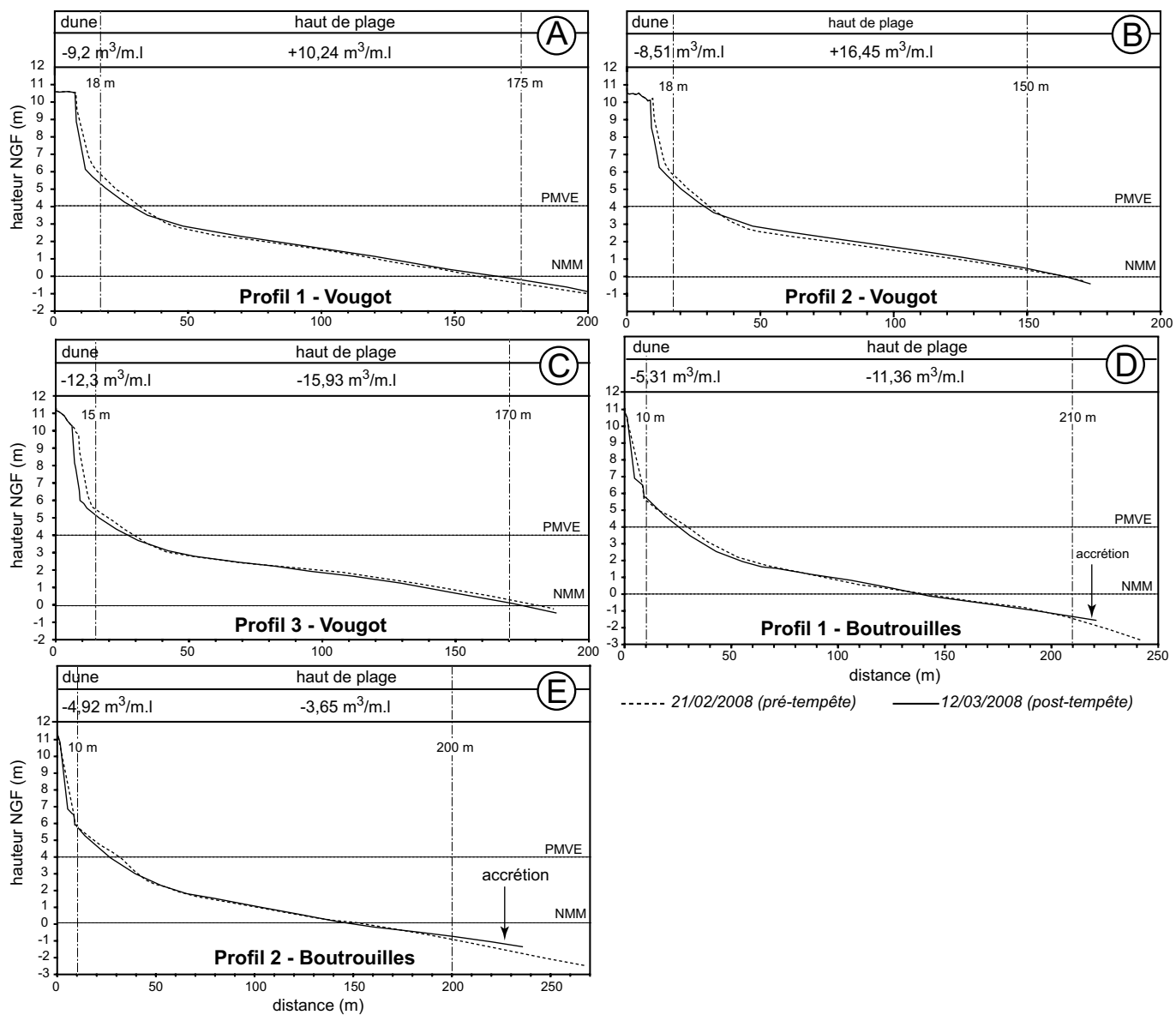

21/02/2008 (pré-tempête) — 12/03/2008 (post-tempête)

Figure 6 : Profils de plage/dune effectués avant et après la tempête du 10 mars 2008 sur les plages du Vougot et de Boutrouilles

Les volumes sédimentaires mentionnés en haut de figure sont calculés sur deux sections du profil (la dune stricto sensu et la haute plage intertidale), dont les limites, en terme de distance, sont données par les valeurs métriques recoupant l'enveloppe de profils.

Beach/dune profile measurements realized before and after the 10th of March storm

Sediment budgets mentioned on the top of the graphs have been calculated on two different sections of the profile (the dune and the upper tidal beach), delimited in terms of distance by metric values mentioned on the envelope of profiles.

\section{L'épisode du 10 mars 2008 dans l'évolution pluriannuelle des dunes}

Replacée dans l'évolution pluriannuelle du cordon dunaire de la plage du Vougot, la tempête du 10 mars apparaît comme un évènement exceptionnel tant les volumes érodés au niveau des trois profils ont été importants (fig. 8, 9 et 10). Les trois illustrations indiquent également que trois phases érosives majeures ont précédé cette tempête (en grisé sur les figures). Elles sont presque toujours centrées autour du mois de mars ( 9 mars 2005, 5 mars 2006, 23 mars 2007 et le 12 mars 2008), et sont caractérisées par des niveaux d'eau extrêmes supérieurs à l'altitude du pied de dune (cf. les tempêtes du 11 février 2005, du 3 mars 2006, du 19 mars 2007, et du 10 mars 2008). 


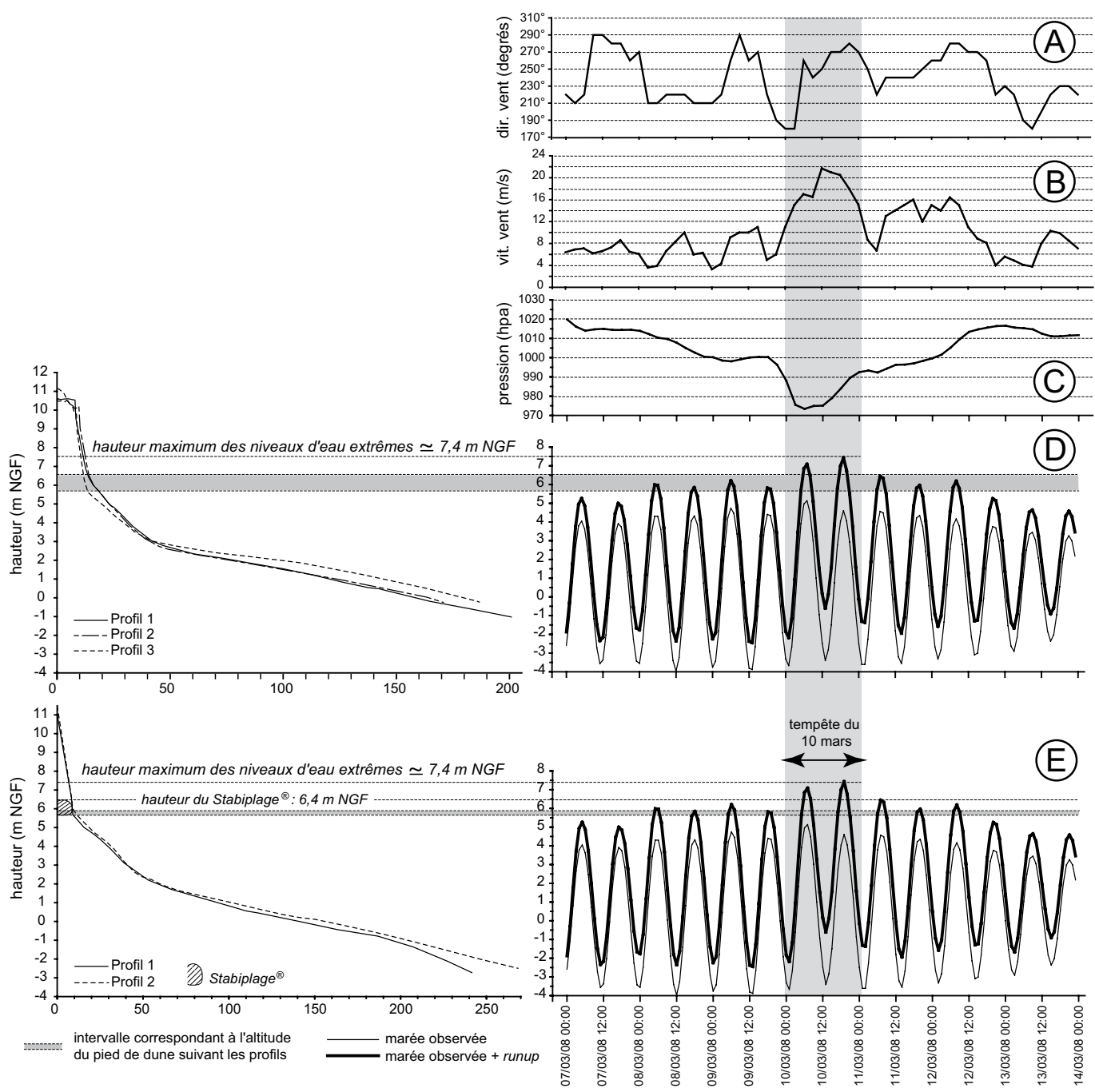

Figure 7 : Conditions météo-atmosphériques et niveaux d'eau extrêmes à la côte observés lors de la tempête du 10 mars 2008

(A) : direction du vent. (B) : vitesse du vent. (C) : pression barométrique. (D) : relation entre niveaux d'eau extrêmes à la côte et altitude du pied de dune pour la plage du Vougot à Guissény. (E) : relation entre niveaux d'eau extrêmes à la côte, altitude du pied de dune, et hauteur du Stabiplage ${ }^{\circledR}$ pour la plage de Boutrouilles à Kerlouan.

Meteo-atmospheric characteristics and extreme water levels recorded during the $10^{\text {th }}$ of March 2008

storm

(A): wind direction. (B): wind speed. $(C)$ : atmospheric pressure. (D): comparison between extreme water levels and height of dune foot on Vougot beach. (E): comparison between extreme water levels and height of dune foot and Stabiplage ${ }^{\circledR}$ on Boutrouilles beach.

D'un point de vue statistique, plus de $80 \%$ des phases d'érosion sont liés à un niveau d'eau supérieur au pied de dune. Pour autant, il n'existe aucune corrélation significative entre le volume de sédiment érodé et le niveau d'eau extrême à la côte $\left(\mathrm{R}^{2}=0,14\right)$ (fig. 11A)

Dans le détail, on peut voir que les trois profils n'observent pas la même évolution. Les profils 1 et 2 , situés le plus à l'est de la plage (fig. 4), évoluent de façon similaire ( $\mathrm{R}^{2} 45 \%$ ) (fig. $\left.11 \mathrm{~B}\right)$; le 

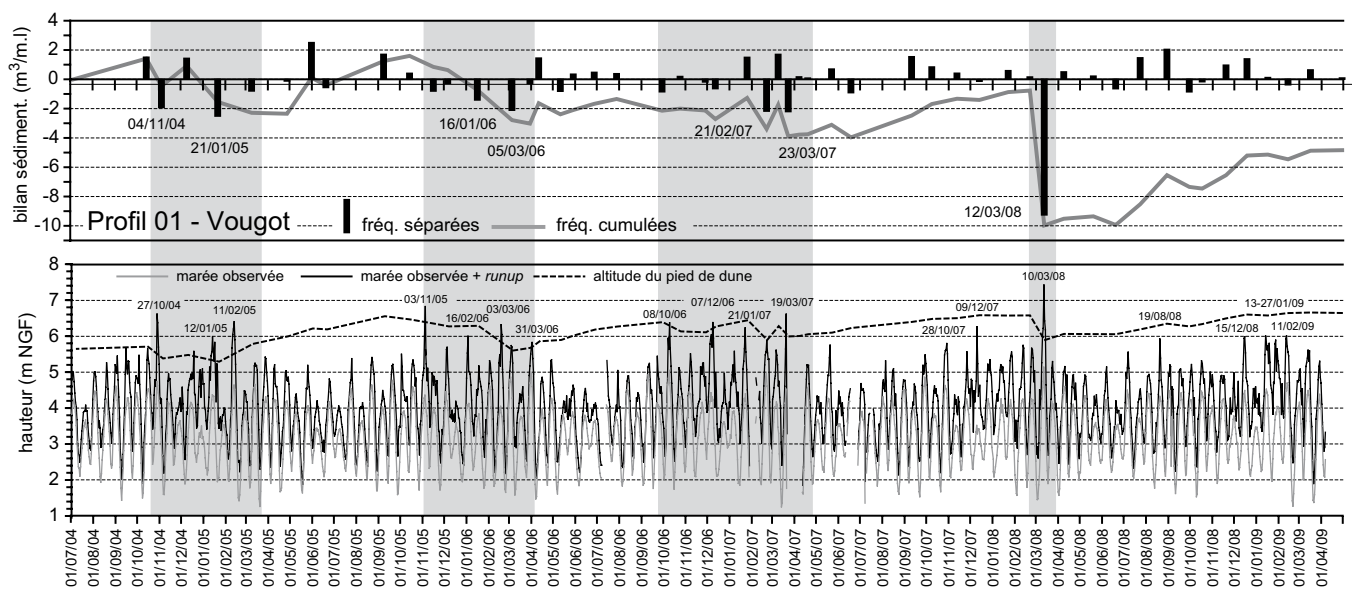

Figure 8 : Évolution du bilan sédimentaire du cordon dunaire de la plage du Vougot au niveau du profil 1, en relation avec les niveaux d'eau extrêmes à la côte sur la période juillet 2004-avril 2009

Sedimentary budget evolution of the dune of Vougot beach regarding profile 1, related to extreme water levels over the period July 2004-April 2009

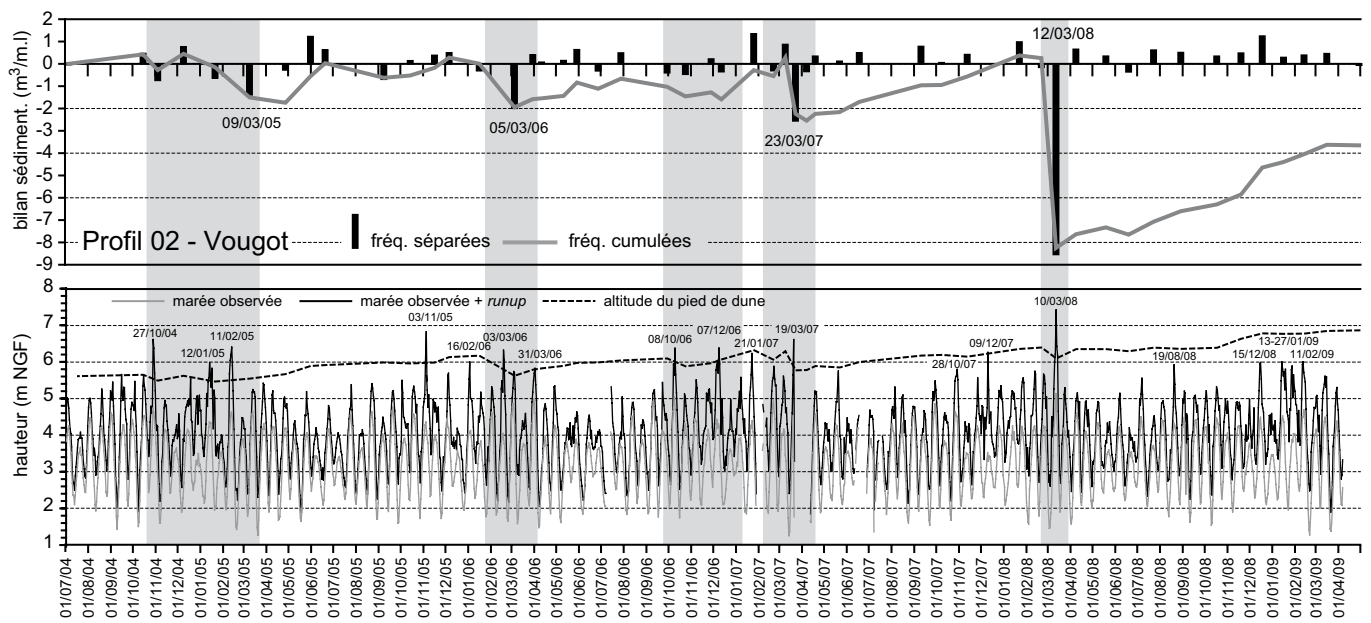

Figure 9 : Évolution du bilan sédimentaire du cordon dunaire de la plage du Vougot au niveau du profil 2, en relation avec les niveaux d'eau extrêmes à la côte sur la période juillet 2004-avril 2009

Sedimentary budget evolution of the dune of Vougot beach regarding profile 2, related to extreme water levels over the period July 2004-April 2009

profil 3, localisé quant à lui plus à l'ouest, montre une évolution distincte des deux premiers $\left(\mathrm{R}^{2}\right.$ $=26 \%$ avec le P01, et $39 \%$ avec le P02) (fig. $11 \mathrm{D}$ et $11 \mathrm{E}$ ). Cette distinction s'exprime particulièrement bien durant les périodes qui suivent les phases érosives notables, à l'exemple de celles qui ont succédé aux tempêtes des mois de mars 2007 et 2008. Durant ces périodes, si les phénomènes de régénération du cordon dunaire sont nettement visibles au niveau des profils 1 et 2 , on s'aperçoit que l'adaptation ${ }^{5}$ de la dune aux nouvelles conditions morphodynamiques ne se fait pas

5. Nous préférons utiliser le terme « d'adaptation » à celui de « résilience » car il est peu probable que ces phénomènes d'alimentation permettent à la dune de retrouver son profil initial avant tempête. 


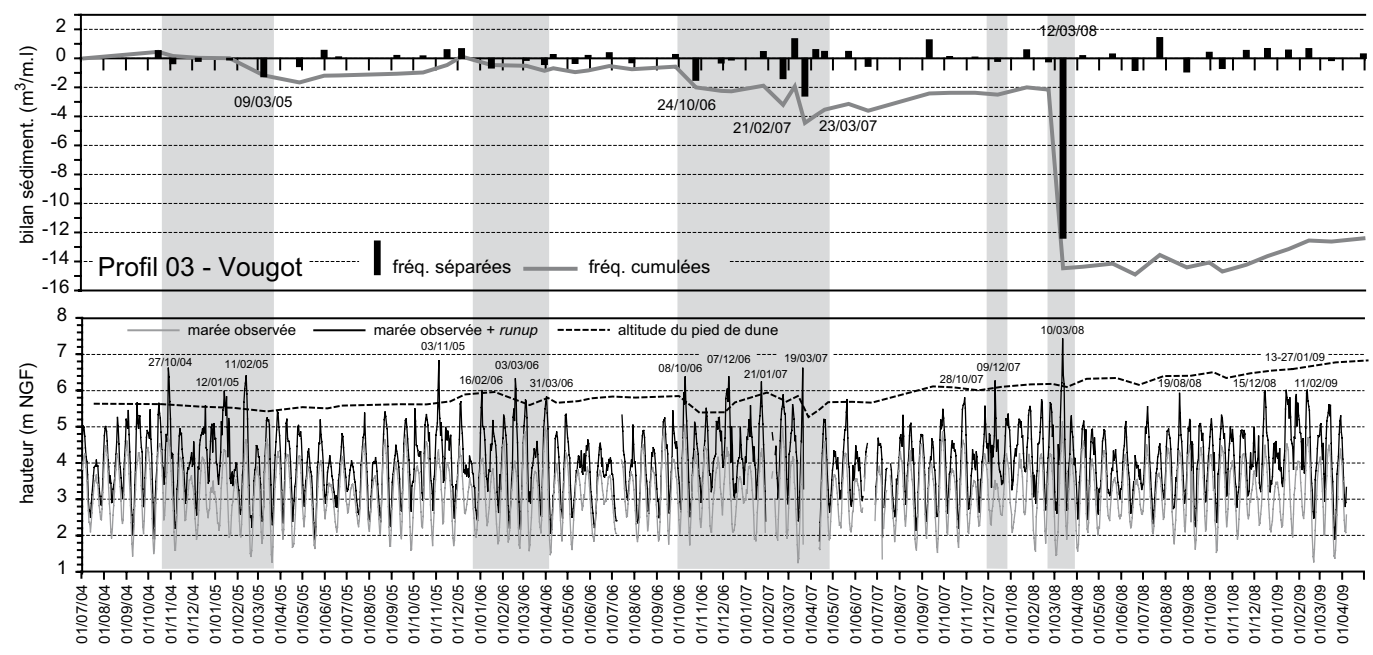

Figure 10 : Évolution du bilan sédimentaire du cordon dunaire de la plage du Vougot au niveau du profil 3, en relation avec les niveaux d'eau extrêmes à la côte sur la période juillet 2004-avril 2009

Sedimentary budget evolution of the dune of Vougot beach regarding profile 3, related to extreme water levels over the period July 2004-April 2009

aussi efficacement au niveau du profil 3. Dans le même temps, durant ces phases de régénération on peut voir que pour les profils 1 et 2, les hauts niveaux d'eau enregistrés du 28 octobre 2008 au 9 décembre 2007, et du 15 décembre 2008 au 11 février 2009, n'entraînent pas d'érosion car le pied de dune s'est largement exhaussé. Là encore, ceci est moins net au niveau du profil 3.

Les effets de la tempête du 10 mars dans l'évolution pluriannuelle du cordon dunaire de la plage de Boutrouilles sont beaucoup moins marqués qu'au Vougot (fig. 12 et 13). Ceci est du à la présence du Stabiplage ${ }^{\circledR}$ qui minimise les processus d'érosion dès lors que le pied de dune est fixé. Ceci étant, une bonne relation entre les niveaux d'eau extrêmes et les phénomènes d'érosion s'observe aussi dans ce cas. Pour les quatre épisodes tempétueux du 8 décembre 2006, du 23 mars 2007, du 13 décembre 2007, et du 12 mars 2008, les niveaux d'eau ayant dépassé et/ou se situant très proche du sommet du Stabiplage ${ }^{\circledR}$ ont entraîné une érosion de la dune.

Les figures 12 et 13 montrent également que le pied de dune est très souvent submergé; à l'exception des périodes estivales, les hauts niveaux d'eau atteignent fréquemment la base du Stabiplage ${ }^{\circledR}$. Cet ouvrage protège donc efficacement le cordon dunaire contre l'érosion marine. Toutefois, comme l'ont montré J.-C. Cariolet et al. (2008), il conditionne le fonctionnement du haut de plage en accentuant les effets réfléchissants de la houle qui entraînent à leur tour le départ de sédiments en haut de plage.

\section{Discussion}

Si les enseignements de la tempête du 10 mars 2008 ont permis de constater que les principes fondamentaux relatifs aux processus d'érosion des dunes pouvaient s'appliquer à nos deux sites d'étude, quelques particularités demeurent. Elles sont liées à l'action des forçages naturels et/ou anthropiques qui donne une spécificité propre à chacune des deux plages.

Le rôle des niveaux d'eau extrêmes dans les processus morphogéniques étudiés apparaît comme l'élément déterminant de l'érosion des dunes. Ces observations vont dans le même sens que les résultats acquis dans le cadre des études de probabilité réalisées par J. van de Graaff (1986) et C. den Heijer et al. (2008). De même, il a été démontré que la hauteur des houles n'intervenait que 

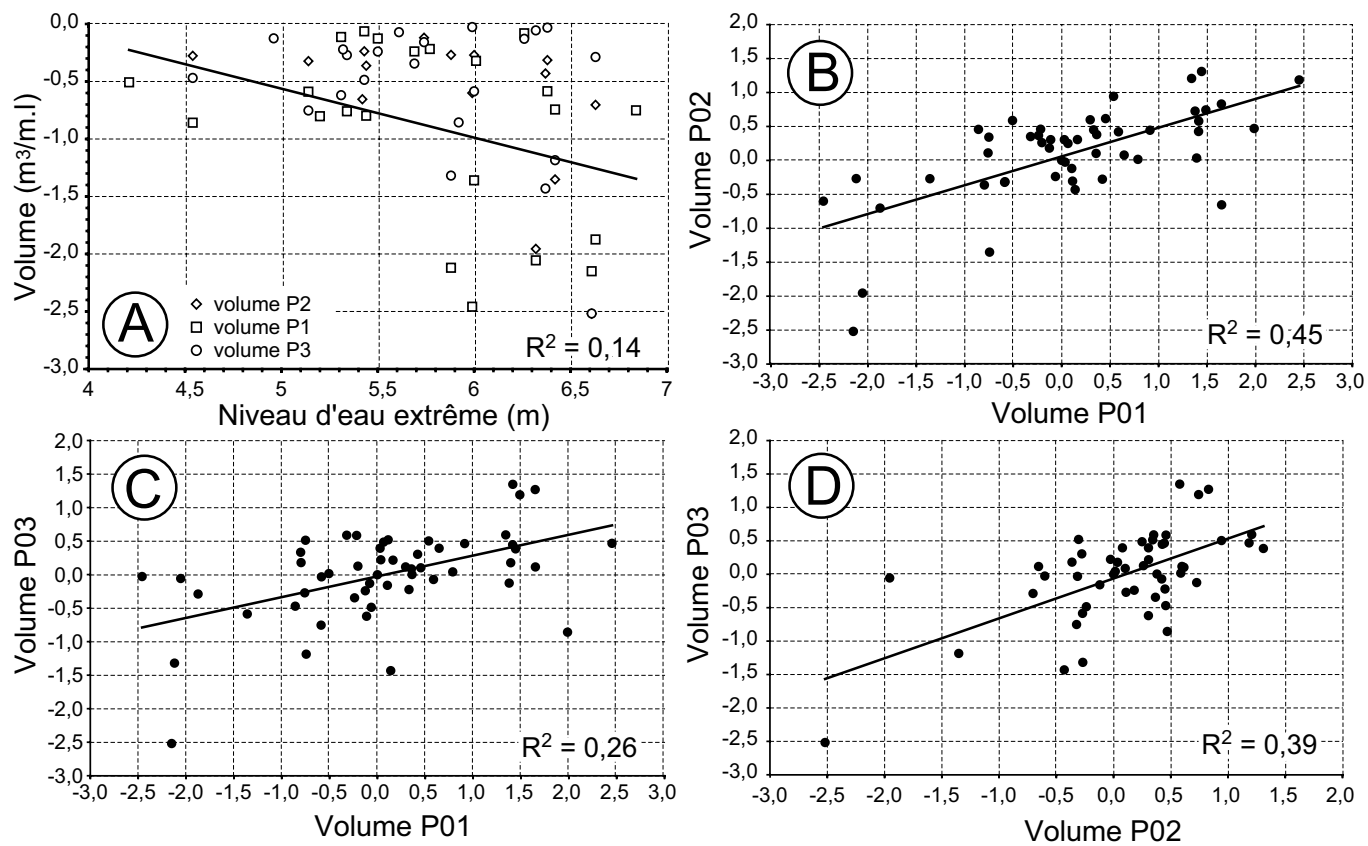

Figure 11 : A : corrélation entre les volumes de sable perdus par le cordon dunaire du Vougot au niveau des trois profils et la hauteur des niveaux d'eau extrêmes. B : corrélation entre les bilans sédimentaires de la dune du Vougot pour les profils 1 et 2 . C : corrélation entre les bilans sédimentaires de la dune du Vougot pour les profils 1 et 3. D : corrélation entre les bilans sédimentaires de la dune du Vougot pour les profils 2 et 3.

A: correlation between sand volume eroded from the dune of Vougot beach (regarding the three profiles) and extreme water level height. B: correlation between sediment budgets of the dune of Vougot beach regarding profiles 1 and 2. C: correlation between sediment budgets of the dune of Vougot beach regarding profiles 1 and 3. D: correlation between sediment budgets of the dune of Vougot beach regarding profiles 2 and 3.

très faiblement dans les processus érosifs. Là encore, l'analyse pluriannuelle des tempêtes, et de leurs effets sur le bilan sédimentaire des cordons dunaires, a montré que ce paramètre ne pouvait à lui seul expliquer l'érosion des dunes. Comme nous l'avons montré, il faut que les fortes houles soient combinées à une marée de vive-eau pour que l'érosion agisse (la tempête du 10 mars en est par ailleurs un parfait exemple, cf. J.-M. Cariolet et al., ce volume). La forme du profil initial avant tempête joue également un rôle important. Comme l'attestent plus particulièrement les résultats obtenus pour la plage du Vougot, les tempêtes du mois de mars ont été pointées comme étant les plus morphogènes comparées à celles de la fin de l'automne, début de l'hiver. Ceci s'explique par le fait qu'au sortir de la période d'alimentation estivale, le système pied de dune/haut de plage est suffisamment exhaussé pour « encaisser » l'attaque des premières houles de tempêtes hivernales. À l'inverse, le profil surbaissé du haut de plage/pied de dune à la fin de l'hiver renforce l'action érosive des tempêtes printanières. Ainsi, comme l'ont montré plusieurs auteurs, la morphologie dunaire, et à travers elle l'ensemble des processus qui agit sur les échanges sédimentaires entre la plage et la dune, contrôle en partie l'action érosive des tempêtes (Short et Hesp, 1982; Davidson-Arnott et Law, 1990 ; Sherman et Bauer, 1993; Morton, 2002 ; Aagaard et al., 2004 ; Battiau-Queney, 2004 ; Ruz et Meur-Ferec, 2004, Saye et al., 2005; Anthony et al., 2006; McLean et Shen, 2006; Pye et Blott, 2008; Sabatier et al., 2009). La fréquence et la durée des épisodes tempétueux contrôlent aussi la résilience du cordon dunaire, et à travers elle la forme du profil de dune avant tempête. M. Ruz et C. Meur-Ferec (2004), A. Vespremeanu-Stroe et L. Preoteasa (2007), et S. Quartel et 


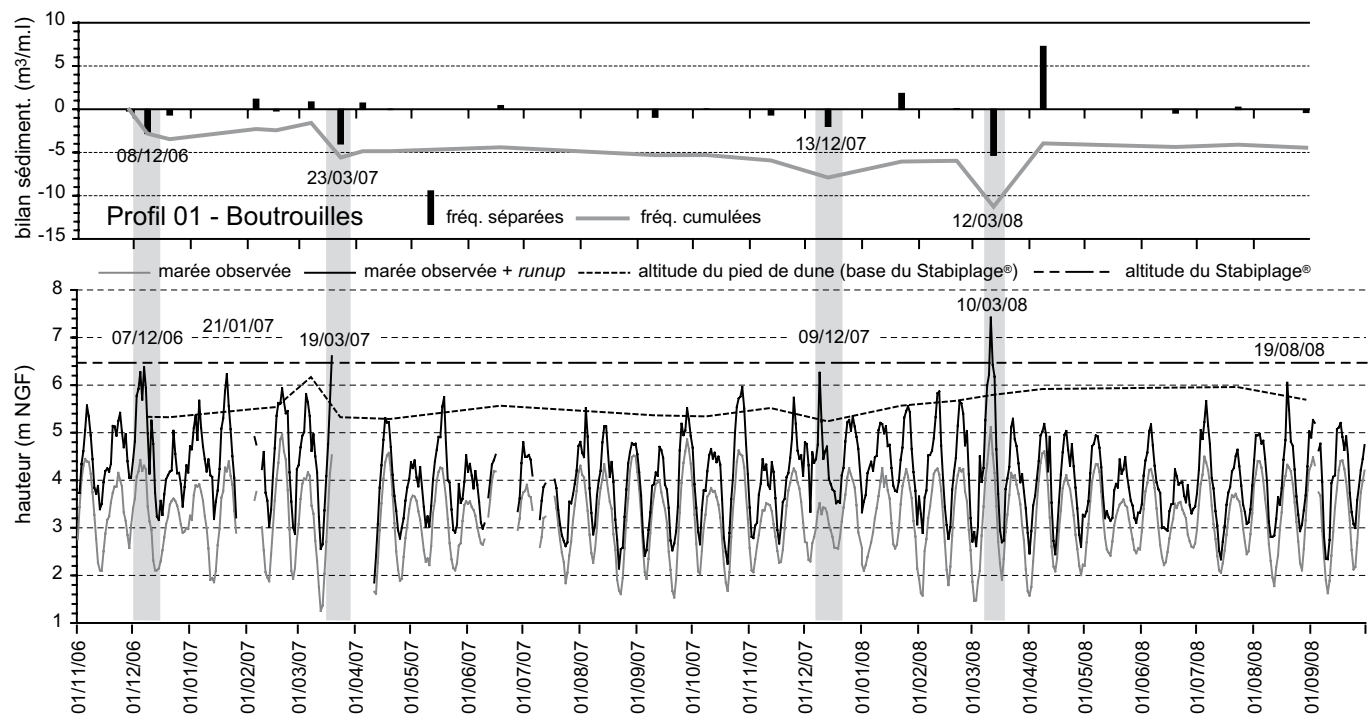

Figure 12 : Évolution du bilan sédimentaire du cordon dunaire de la plage de Boutrouilles au niveau du profil 1 , en relation avec les niveaux d'eau extrêmes à la côte sur la période novembre 2006-septembre 2008

Sedimentary budget evolution of the dune of Boutrouilles beach regarding profile 1, related to extreme water levels over the period November 2006-September 2008

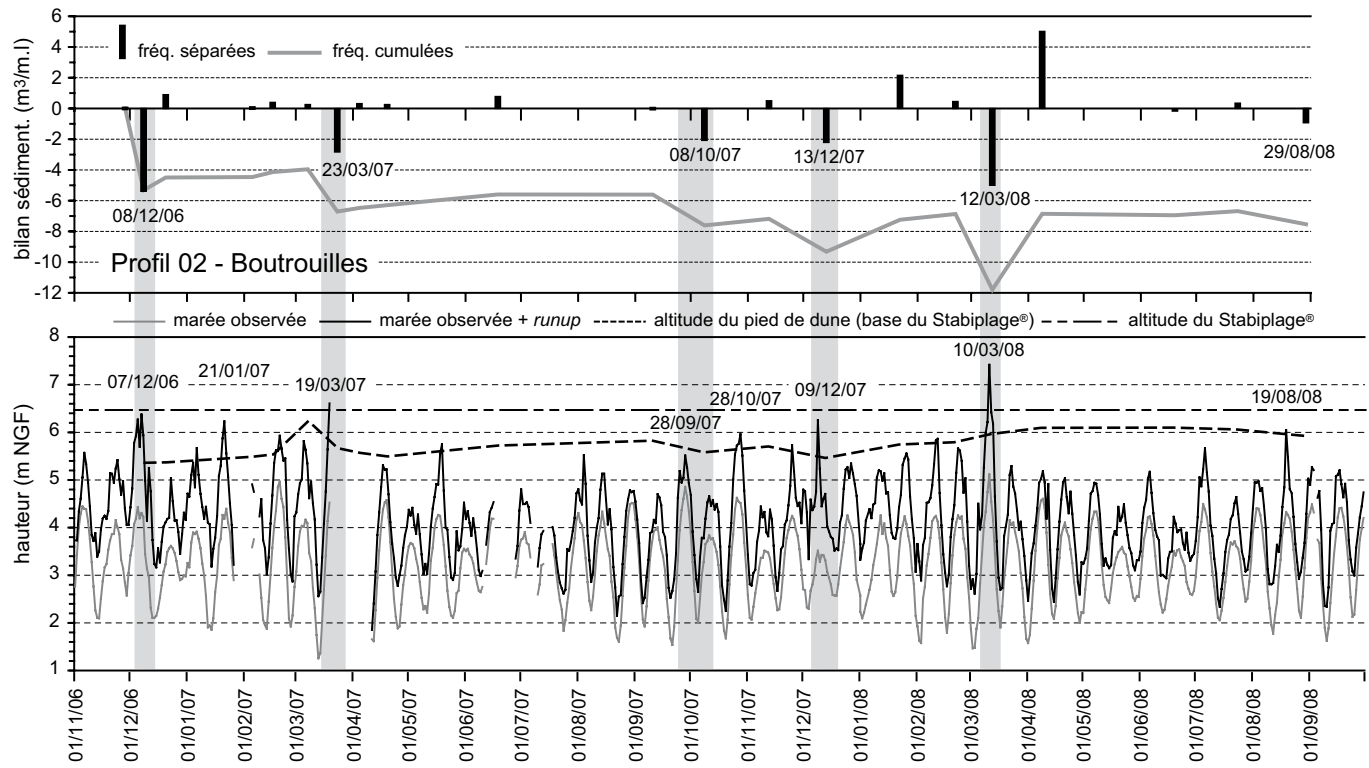

Figure 13 : Évolution du bilan sédimentaire du cordon dunaire de la plage de Boutrouilles au niveau du profil 2, en relation avec les niveaux d'eau extrêmes à la côte sur la période novembre 2006-septembre 2008

Sedimentary budget evolution of the dune of Boutrouilles beach regarding profile 2, related to extreme water levels over the period November 2006-September 2008 
al., (2008) ont démontré que plus les tempêtes sont longues et rapprochées, plus la capacité du système littoral à se régénérer diminue car les échanges sédimentaires entre la plage et la dune ne se font plus. La plage dans son ensemble devient alors plus vulnérable à l'action des tempêtes. Ces processus caractérisent particulièrement bien les hivers 2004-2005, 2005-2006 et 2006-2007 où l'on voit, à partir des données acquises pour la plage du Vougot, que la fréquence élevée des tempêtes a joué dans l'affaiblissement du cordon dunaire. Durant ces périodes, la faiblesse des apports sédimentaires s'est traduite par un abaissement du pied de dune, renforçant ainsi l'action érosive des tempêtes printanières des années 2005, 2006 et 2007. À l'inverse, la bonne régénération du cordon dunaire durant les hivers 2007-2008 et 2008-2009 a permis de protéger la dune contre les tempêtes des mois de mars 2008 et 2009, à l'exception bien sûr de l'épisode du 10 mars 2008 qui revêt, comme on l'a dit plus haut, un caractère exceptionnel. Les processus d'érosion observés montrent que le principe d'ajustement du profil d'équilibre décrit par P. Bruun (1962) peut s'appliquer dès lors que les transferts sédimentaires transversaux ne sont pas influencés par d'autres dynamiques. Ainsi, pour les profils 1 et 2 de la plage du Vougot, les volumes érodés à la dune semblent bien alimenter la haute plage intertidale, à la différence de la radiale 3, pour laquelle les deux sections du profil sont déficitaires (fig. 6). Lorsque l'on observe les résultats obtenus à l'échelle pluriannuelle pour les phases érosives majeures, à l'exception de quelques cas, ce fonctionnement reste le même (fig. 14). Nous interprétons ces différences comme étant liées à l'action des transferts longitudinaux qui agissent de façon plus efficace et systématique à l'ouest de la plage du Vougot (Suanez et al., 2010). Ainsi, au niveau du profil 3, le volume de sable érodé à la dune est très rapidement exporté en direction du Zorn alors que ces déplacements prennent plus de temps au niveau des profils 1 et 2 . Pour ces deux radiales, le principe d'ajustement du profil d'équilibre d'après tempête est alors plus lisible.
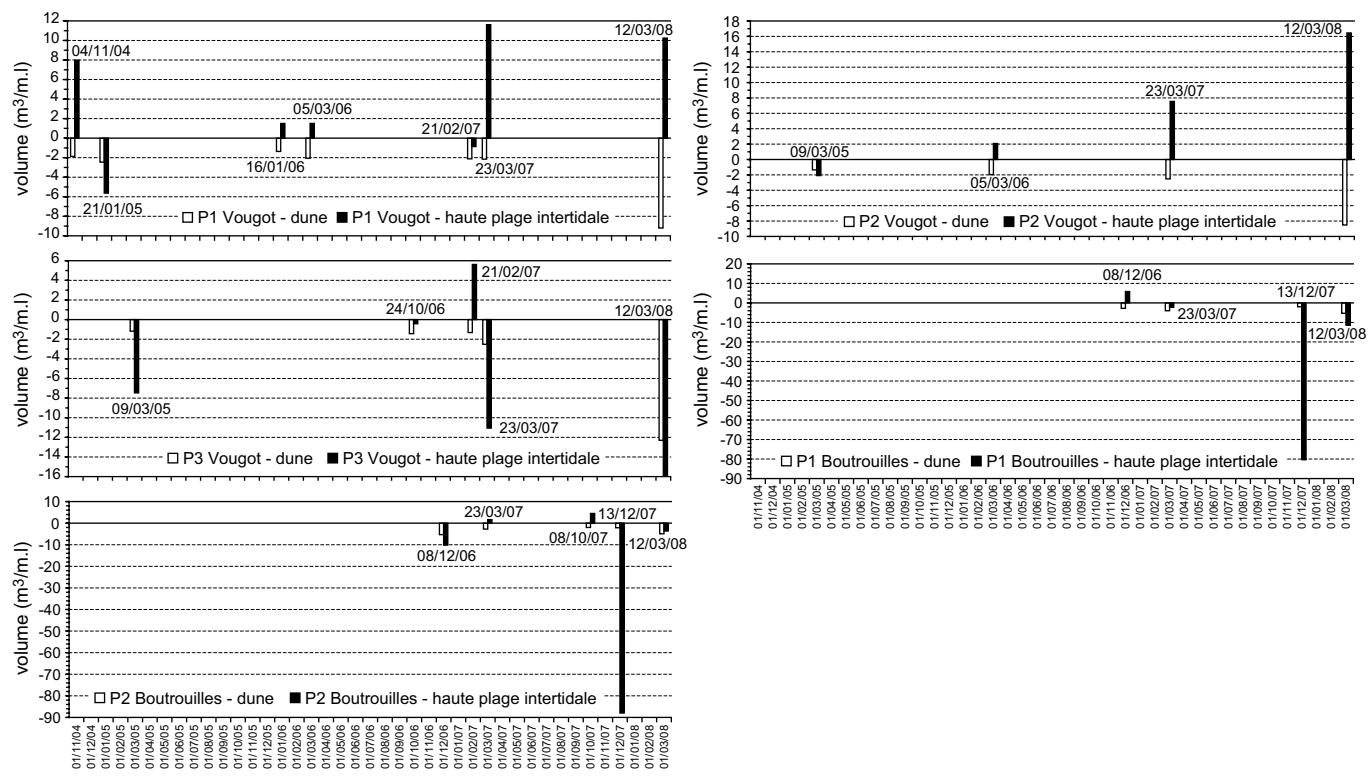

Figure 14 : Bilans sédimentaires (en $\mathrm{m}^{3} / \mathrm{m} . \mathrm{l}$ ) de la dune et de la haute plage intertidale des plages du Vougot et de Boutrouilles pour les phases érosives majeures enregistrées depuis 2004

Sediment budgets (in $\left.\mathrm{m}^{3} / \mathrm{l} . \mathrm{m}\right)$ of the dune and the upper intertidal beach of Vougot and Boutrouilles beaches regarding the major erosive phases since 2004 
Serge Suanez, Jean-Marie Cariolet

Pour la plage de Boutrouilles, les choses apparaissent beaucoup plus compliquées. Les données acquises sur la tempête du 10 mars ont montré que l'ensemble du profil de plage/dune était déficitaire (fig. 6). De même, les résultats acquis à l'échelle pluriannuelle indiquent que la majorité des tempêtes se sont soldées par un déficit sédimentaire au niveau des deux sections du profil (fig. 14) - à l'exemple du relevé effectué le 13 décembre 2007 après la tempête du 7 décembre 2007. L'absence de phasage entre l'érosion de la dune et l'alimentation de la haute plage intertidale s'explique vraisemblablement par l'action efficace des courants de retour générés par la présence du Stabiplage ${ }^{\circledR}$. Comme nous l'avons souligné plus haut, J.-M. Cariolet et al., 2008 ont montré que la réflexion de la houle contre l'ouvrage favorisait l'érosion du haut de plage tout en accentuant l'exportation de ce matériel vers le bas de plage. Comme l'ont indiqué les mesures effectuées le 12 mars 2008, les phénomènes d'accrétion visibles au-delà d’une distance de 200 m par rapport au pied de dune illustrent probablement l'action des ces transits transversaux (fig. 6D et 6E).

\section{Conclusion}

D'un point de vue morphogénique, l'impact de la tempête du 10 mars 2008 sur l'érosion des dunes du nord Finistère revêt un caractère exceptionnel à l'échelle des vingt dernières années ; en terme de comparaison, il faut remonter aux tempêtes de l'hiver 1989-1990 qui avaient été aussi particulièrement dévastatrices (Garabœuf, 1990). L'action érosive a été d'autant plus exacerbée, et aura des effets d'autant plus durables, que ces deux plages connaissent depuis plusieurs décennies un déficit sédimentaire chronique. Ceci étant, depuis le printemps 2008 des phénomènes de ré-engraissement de la dune du Vougot ont été observés. Ces nouveaux apports sableux ont littéralement "masqué » la falaise d'érosion au niveau des profils 1 et 2, ou entraîné la construction d'un chapelet de dunes embryonnaires en haut de plage comme au niveau du profil 3. Ainsi, si l'on replace l'ensemble des dynamiques observées au sein des modèles conceptuels (Ruz, 2004), l'évolution à long terme des cordons dunaires des plages du Vougot et de Boutrouilles correspondrait au scénario (D) du modèle de N. P. Psuty (1988, 1992), caractérisé par un bilan sédimentaire déficitaire de l'ensemble du système plage/dune. Dans ce contexte, l'auteur indique que l'érosion des dunes bordières est avant tout liée aux effets de « débordement de tempête », et que par rétroaction, l'abaissement de plus en plus accru du profil de plage favorise l'attaque de plus en plus fréquente de la dune. Ces processus illustrent parfaitement le fonctionnement du système plage/dune de la plage du Vougot même si comme on l'a vu, des phénomènes de régénération du cordon dunaire peuvent agir à l'échelle saisonnière (essentiellement durant l'été). Si l'on se rapporte au modèle de P. A Hesp (1988, 2002), ces phénomènes de ré-engraissement n'agissent que sur du court terme; ils dépendent généralement de la disponibilité en sédiment et de la fréquence des évènements érosifs à l'échelle saisonnière. Pour autant, nous ne pouvons en aucun cas parler de résilience du cordon dunaire car cette alimentation n'a pas entraîné une avancée du front de dune de telle sorte qu'il retrouve la position qu'il observait avant la tempête. Pour la plage de Boutrouilles, la présence d'un ouvrage de protection en pied de dune a permis de stopper « pour un temps » le recul de la dune. Toutefois, comme l'a montré la tempête du 10 mars, le dimensionnement de la structure et/ou son positionnement présentent des insuffisances car il peut-être submergé lors d'évènements exceptionnels. De plus, comme l'ont indiqué J.-M. Cariolet et al. (2008), l'érosion chronique de la plage, et avec elle l'abaissement de son profil, pourrait à terme déstabiliser le Stabiplage ${ }^{\circledR}$ qui ne jouerait alors plus correctement son rôle de protection de la dune.

\section{Remerciements}

Ce travail a été financé par la Région Bretagne dans le cadre d'un contrat Nature; il a également bénéficié de financements de la commune de Guissény dans le cadre de Natura 2000 au titre de la conservation et de l'entretien des milieux dunaires (objectif A3). Nous tenons à remercier R. Magne 
du SHOM pour la mise à disposition des données de houles. Nous remercions également Guy Wöppelmann, responsable du projet SONEL, pour la mise à disposition des données marégraphiques observées. Merci à Laurence David (Géomer) pour sa contribution dans la réalisation de certaines cartes.

\section{Bibliographie}

Aagard T., Davidson-Arnott R., Greenwood B., Nielsen J., 2004. Sediment supply from shoreface to dunes: linking sediment transport measurements and long-term morphological evolution, Geomorphology, vol. $60, n^{\circ} 1-2$, p. $205-224$.

Anthony E. J., Vanhee S., Ruz M.-H., 2006. Short-term beach-dune sand budgets on the north sea coast of France: Sand supply from shoreface to dunes, and the role of wind and fetch, Geomorphology, vol. 81, $n^{\circ} 3-4$, p. 316-329.

Battiau-Queney Y., 2004. Haut de plage et front dunaire : enregistrement morphologique de la dynamique éolienne et marine dans un système macrotidal, Bulletin de l'Association des Géographes Français, $\mathrm{n}^{\circ} 3$, p. 393-404.

Battjes J.A., 1974. Surf similarity. Proceeding of the $14^{\text {th }}$ Coastal Engineering Conference, ASCE, p. 466479.

Benavente J., Del Río L., Gracia F.J., Martínez-del-Pozo J.A., 2006. Coastal flooding hazard related to storms and coastal evolution in Valdelagrana spit (Cadiz Bay Natural Park, SW Spain), Continental Shelf Research, vol. 26, n 9, p. 1061-1076.

Bodéré J.-C., Hallégouët B., Piriou N., 1983. Schéma de protection contre la mer du littoral finistérien, Communes de Guissény et de Plouguerneau, Zone du Curnic. Rapport d'étude, Faculté des Lettres et des Sciences Sociales, Université de Bretagne Occidentale, 81 p. (n.p.)

Bruun P., 1962. Sea level rise as a cause of shore erosion, Journal of Waterway and Harbours Division, vol. $88, n^{\circ} 1-3$, p. 117-130.

Cariolet J.-M., Costa S., Caspar R., Ardhuin F., Magne R., Goasguen G., Aspects météo-marins de la tempête du 10 mars 2008 en Atlantique et en Manche, Norois, (ce volume).

Cariolet J.-M., Suanez S., 2009. Approche méthodologique pour une cartographie du risque de submersion des côtes basses, La Houille Blanche, $\mathrm{n}^{\circ}$ 2, p. 52-58.

Cariolet J.-M., Suanez S., Carol F., Magne R., 2008. Evaluation de la technique STABIPLAGE ${ }^{\circledR}$ mise en place sur deux plages du Finistère : les Sables Blancs à Plobannalec-Lesconil-Loctudy, et Boutrouilles à Kerlouan, Actes du colloque des X ${ }^{\text {es }}$ Journées Nationales Génie Civil Génie Côtier, 14-16 oct. 2008, Nice Sophia-Antipolis, D. Levacher, P. Gaufres (dir.), p. 201-210 [http://wwww.paralia.frijngcgc/10_19_cariolet.pdf].

Carter R. W. G., Stone G. W., 1989. Mechanisms associated with the erosion of sand dune cliffs, Magilligan, Northern Ireland, Earth Surface Processes and Landforms, vol. 14, n 1, p. 1-10.

Carter R. G. W., Hesp P. A., Nordstrom K. F., 1990. Erosional landforms in coastal dunes, dans Nordstrom K., Psuty N., Carter B. (dir.), Coastal dunes. Form and process, Edit. John Wiley \& Sons, England, p. 217-250.

Carter R. W. G., 1980. Vegetation stabilisation and slope failure of eroding sand dunes, Biological Conservation, vol. 18, n² 2, p. 117-122.

den Heijer C., van de Graaff J., van Gelder P., 2008. Probabilistic sensitivity of dune erosion calculations, $4^{\text {th }}$ International Symposium on Flood Defence (Toronto, Ontario, Canada, May 6-8, 2008), Institute for Catastrophic Loss Reduction (ICLR), Toronto (Ontario), Canada, p. 69.1-69.8.

Davidson-Arnott R. G. D., Law M. N., 1990. Seasonal patterns and controls on sediment supply to coastal foredunes, Long Point, Lake Erie, dans Nordstrom K., Psuty N., Carter B. (dir.), Coastal dunes. Form and process, Edit. John Wiley \& Sons, England, p. 177-200.

Edelman, T., 1968. Dune erosion during storm conditions, Proceeding of the 11 th Conference on Coastal Engineering, ASCE, vol. 1, p. 719-722.

—, 1972. Dune erosion during storm conditions, Proceeding of the 13th Conference on Coastal Engineering, ASCE, vol. 2, p. 1305-1311. 
Erchinger H. F., 1992. Conservation of barrier dunes as a smooth, natural method of coastal protection on the East Friesian Islands, Germany, dans Carter R. W. G., Curtis T. G. F., Sheehy-Skeffington M. J. (dir.), Coastal dunes. Geomorphology, Ecology and Management for Conservation, Proc. $3^{\text {rd }}$ European Dune Congress, Balkema, Rotterdam, p. 389-396.

Erikson L. H., Larson M., Hanson H., 2007. Laboratory investigation of beach scarp and dune recession due to notching and subsequent failure, Marine Geology, vol. 245, n 1-4, p. 1-19.

Fisher J. S., Overton M. F. (1984) - Numerical model for dune erosion due to wave uprush, Proceedings of the $19^{\text {th }}$ Coastal Engineering Conference, ASCE, p. 1553-1558.

Garabceuf M.-F., 1990. Le littoral guissénien, Mémoire de DEA, Université de Bretagne Occidentale, 73 p. et annexes.

Gómez-Pina G., Muńoz-Pérez J. J., Ramírez J. L., Ley C., 2002. Sand dune management problems and techniques, Spain, Journal of Coastal Research, Special Issue n 36, p. 325-332.

Guilcher A., Hallégouët B., 1991. Coastal dunes in Brittany and their management, Journal of Coastal Research, vol. 7, n 2, p. 517-533.

Hallégouët B., 1978. Lévolution des massifs dunaires du pays de Léon, Penn ar Bed, vol. 11, n 95, p. 417 430.

—, 1971. Le Bas-Léon (Finistère, France). Étude géomorphologique, Thèse de 3e cycle, Université de Bretagne Occidentale, $260 \mathrm{p}$.

Hesp P. A., 1988. Surfzone, beach and fordune interactions on the Australian southeast coast, Journal of Coastal Research, Special Issue n 3, p. 325-332.

-, 2002. Foredunes and blowouts: initiation, geomorphology and dynamics, Geomorphology, vol. 48, nº 1-3, p. $245-268$.

Kriebel D. L., 1985. Numerical simulation of time-dependant beach and dune erosion, Coastal Engineering, vol. $9, \mathrm{n}^{\circ} 3$, p. 221-245.

—, 1986. Verification study of a dune erosion model, Shore \& Beach, vol. 54, n 3, p. 13-21.

Larson M., Erikson L., Hanson H., 2004. An analytical model to predict dune erosion due to wave impact, Coastal Engineering, vol. 51, n 8-9, p. 675-696.

Louisse C.J., van der Meulen F., 1991. Future coastal defence in the Netherland: strategies for protection and sustainable development, Journal of Coastal Research, vol. 7, n 4, p. 1027-1041.

McLean R., Shen J. S., 2006. From foreshore to fordune: Foredune development over the last 30 years at Moruya beach, New South Wales, Australia, Journal of Coastal Research, vol. 22, nº 1, p. 28-36.

Morton R. A., 2002. Factors controlling storm impacts on coastal barriers and beaches - a preliminary basis for real-time forecasting, Journal of Coastal Research, vol. 18, n³ 3, p. 486-501.

Overton M. F., Fisher J. S., 1988. Laboratory investigation of dune erosion, Journal of Waterway, Port, Coastal, and Ocean Engineering, vol. 114, n 3, p. 367-373.

Psuty N. P., 1988. Sediment budget and dune/beach interaction, Journal of Coastal Research, Special Issue $\mathrm{n}^{\circ} 3$, p. 1-4.

-, 1992. Spatial variation in coastal Foredune development, dans Carter R. W. G., Curtis T. G. F., SheehySkeffington M. J. (dir.), Coastal dunes. Geomorphology, Ecology and Management for Conservation, Proc. $3^{\text {rd }}$ European Dune Congress, Balkema, Rotterdam, p. 3-13.

Pye K., Neal A., 1994. Coastal dune erosion at Formby Point, north Merseyside, England: causes and mechanisms, Marine Geology, vol. 119, n 1-2, p. 39-56.

Pye K., Blott S. J., 2008. Decadal-scale variation in dune erosion and accretion rates: an investigation of the significance of changing storm tide frequency and magnitude on the Sefton coast, UK, Geomorphology, vol. 102, n 3-4, p. 652-666.

Quartel S., Kroon A., Ruessink B. G., 2008. Seasonal accretion and erosion patterns of a microtidal sandy beach, Marine Geology, vol. 250, n 1-2, p. 19-33.

Ruggiero P., Komar P. D., McDouglas W. G., Marra J. J., Beach R. A., 2001. Wave runup, extreme water levels and erosion of properties backing beaches, Journal of Coastal Research, vol. 17, $\mathrm{n}^{\circ}$ 2, p. 407-419.

Ruessink B. G., Jeuken M. C. J. L., 2002. Dunefoot dynamics along the Dutch coast, Earth Surface Processes Landforms, $n^{\circ} 27$, p. 1043-1056. 
Ruz M.-H., Meur-Ferec C., 2004. Influence of high water levels on aeolian sand transport: upper beach/ dune evolution on a macrotidal coast, Wissant Bay, northern France, Geomorphology, vol. 60, n 1-2, p. 73-87.

Ruz M.-H., 2004. Formation et évolution des dunes bordières : modèles conceptuels, Bulletin de l'Association des Géographes Français, n 3, p. 383-392.

Sabatier F., Anthony E., Héquette A., Suanez S., Musereau J., Régnauld H., Ruz M., 2009. Morphodynamics of beach/dune systems: examples from the coast of France, Géomorphologie : relief, processus, environnement, $\mathrm{n}^{\circ}$ 1, p. 3-22.

SAllenger A. H. Jr., 2000. Storm impact scale for barrier islands, Journal of Coastal Research, vol. 16, $n^{\circ} 3$, p. 890-895.

Sallenger A. H. Jr., Krabill W., Brock J., Swift R., Manizade S., Stockdon H., 2002. Sea-cliff erosion as a function of beach changes and extreme wave runup during the 1997-1998 El Niño, Marine Geology, vol. $187, n^{\circ} 3-4$, p. 279-297.

Saye S. E., van der Wal D., Pye K., Blott S. J., 2005. Beach-dune morphological relationships and erosion/ accretion at five sites in England and Wales using LIDAR data, Geomorphology, vol. 72, n 1-4, p. 128-155.

Sherman D. J., Bauer B. O., 1993. Dynamics of beach-dune systems, Progress in Physical Geography, vol. $17 \mathrm{n}^{\circ} 4$, p. 413-447.

Short A. D., Hesp P. A., 1982. Wave, beach and dune interactions in southeast Australia, Marine Geology, vol. 48, n³-4, p. 259-284.

Sparfel L., Suanez S., 2007. Le vougot à l'épreuve des flots, Penn ar Bed, nº 199/200, p. 39-49.

Stockdon H. F., Holman R. A., Howd P. A., Sallenger A. H., 2006. Empirical parametrization of setup, swash and runup, Coastal Engineering, vol. 53, $\mathrm{n}^{\circ} 7$, p. 573-588.

Stockdon H. F., Thompson D. M., 2007. Vulnerability of National Park Service Beaches to Inundation during a Direct Hurricane Landfall: Cape Lookout National Seashore, U.S. Geological Survey, Open-File Report 2007-1376, 8 p.

Stockdon H. F., Sallenger A. H., Holman R. A., Howd P. A., 2007. A simple model for the spatiallyvariable coastal response to hurricanes, Marine Geology, vol. 238, n 1-4, p. 1-20.

Stauble D. K., Eiser W. C., Birkemeier W. A., Hales L. Z., Seabergh W. C., 1990. Erosion characteristics of Hurricane Hugo on the beaches of South Carolina, Shore E Beach, vol. 58, n 4, p. 23-36.

Suanez S., Fichaut B., Sparfel L., 2007. Méthode d'évaluation du risque de submersion des côtes basses appliquée à la plage du Vougot, Guissény (Bretagne), Géomorphologie : relief, processus, environnement, ${ }^{\circ} 4$, p. 319-334.

Suanez S., StÉphan P., 2006. Forçages météo-marins et dynamique morphosédimentaire saisonnière des cordons dunaires. Exemple de la baie de Saint-Michel-en-Grève (Côtes d'Armor, Bretagne), Géomorphologie : relief, processus, environnement, $\mathrm{n}^{\circ} 2$, p. 91-110.

Suanez S., Cariolet J.-M., Fichaut B., 2010. Monitoring of recent morphological changes of the dune of Vougot beach (Brittany, France) using differential GPS, Shore E Beach, vol. 78, n 1, p. 37-47.

van de Graaff J., 1977. Dune erosion during a storm, Coastal Enginnering, n 1, p. 99-134.

—, 1986. Probabilistic design of dunes; an example from the Netherlands, Coastal Engineering, vol. 9, n 5 , p. $470-500$.

van der Meulen, T., Gourlay, M. R., 1968. Beach and dune erosion test, Proceeding of the 11 th Conference on Coastal Engineering, ASCE, vol. 1, p. 701-707.

Vellinga P., 1982. Beach and dune erosion during storm surges, Coastal Engineering, vol. 6, n 4, p. 361-387.

—, 1984. Movable-bed modelling law for coastal dune erosion, Journal of Waterway Port Coastal and Ocean Engineering, vol. 11, n 4, p. 495-504.

Vespremeanu-Stroe A., Preteasa L., 2007. Beach-dune interactions on the dry-temperate Danube delta coast, Geomorphology, vol. 86, n³ 3-4, p. 267-282.

Wright L. D., Short A. D., 1984. Morphodynamics variability of surf zones and beaches: a synthesis, Marine Geology, vol. 56, n 1-4, p. 93-118. 